\title{
Article \\ Soil Aeration and Plastic Film Mulching Increase the Yield Potential and Quality of Tomato (Solanum lycopersicum)
}

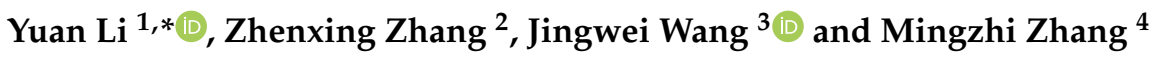

1 Northwest Land and Resources Research Center, Shaanxi Normal University, Xi'an 710119, China

2 Key Laboratory of Vegetation Ecology, Institute of Grassland Science, Northeast Normal University, Ministry of Education, Changchun 130024, China; zhangzx725@nenu.edu.cn

3 College of Resources and Environment, Shanxi University of Finance and Economics, Taiyuan 030006, China; wjw@sxufe.edu.cn

4 Henan Provincial Water Conservancy Research Institute, Zhengzhou 450000, China; sksmingzhiz@hnsl.gov.cn

* Correspondence: liy681@nenu.edu.cn

check for updates

Citation: Li, Y.; Zhang, Z.; Wang, J.; Zhang, M. Soil Aeration and Plastic Film Mulching Increase the Yield Potential and Quality of Tomato (Solanum lycopersicum). Agriculture 2022, 12, 269. https://doi.org/ 10.3390 /agriculture12020269

Academic Editor: Jochen Mayer

Received: 25 January 2022

Accepted: 11 February 2022

Published: 14 February 2022

Publisher's Note: MDPI stays neutral with regard to jurisdictional claims in published maps and institutional affiliations.

Copyright: (c) 2022 by the authors. Licensee MDPI, Basel, Switzerland. This article is an open access article distributed under the terms and conditions of the Creative Commons Attribution (CC BY) license (https:// creativecommons.org/licenses/by/ $4.0 /)$.

\begin{abstract}
Soil aeration and plastic film mulching have been reported to accelerate plant growth and increase fruit yield by improving the rhizosphere soil-air environment. The aim of this study was to investigate plant growth, fruit yield, irrigation water-use efficiency (IWUE) and fruit quality in response to a micro/nano-bubble aeration (MNBA) system and a subsurface artificial air layer aeration system (SAALA) under different treatments. The results indicated that both MNBA and SAALA positively influenced the plant dry weight, fruit yield, IWUE and fruit quality. In comparison with the no aeration treatment, the MNBA treatment increased the dry matter accumulation, fruit yield, IWUE, lycopene content and soluble protein content by $7.1 \%, 9.0 \%, 7.1 \%, 6.2 \%$ and $16.2 \%$, respectively. Plastic film mulching (PFM) significantly improved the total dry weight, fruit yield and IWUE during both seasons. The increased yield in response to soil aeration during autumn was significantly greater than that during spring, and the yield increase in response to PFM was significantly greater in spring than in autumn. Moreover, the nutrition indices in response to both soil aeration and PFM were more significant during spring than autumn. Taking into account costs, efficiency and benefits, the optimal treatment was the MNBA and full (F) PFM combination.
\end{abstract}

Keywords: micro/nano-bubble aeration; subsurface artificial air layer aeration; plastic film mulching; tomato; quality

\section{Introduction}

Oxygen $\left(\mathrm{O}_{2}\right)$ deficiency in agricultural soils under intensive irrigation is a well-known phenomenon, especially in fine-textured, poorly drained, clayey soils [1]. Previous studies have revealed that various physiological processes and components, such as nitrogen metabolism and translocation [2,3], root resistance to disease, photosynthesis, stomatal behaviour [4], relative membrane permeability, hydrogen peroxide activity, malondialdehyde content, pyruvate decarboxylase activity, alcohol dehydrogenase activity, and lactate dehydrogenase activity [5], can be altered under hypoxic stress. Other studies have also demonstrated the negative effects of $\mathrm{O}_{2}$ deficiency in the root zone on plant growth, soil respiration and nutrient uptake [6,7].

Oxygen deficiency in agricultural soils is also enhanced by the use of plastic film mulching (PFM). PFM is largely used in many cultivations because it greatly reduces evaporation, increases the average soil temperature, decreases the diurnal variation in the temperature of the root zone, increases both topsoil moisture and water-use efficiency (WUE) and improves crop yield [8-12]. Mo et al.'s research [13] also found that PFM can effectively improve sunlight reflectance and decrease air flow, weed and pest pressure at the soil surface. In terms of the air phase, PFM inevitably blocks the air exchange between the soil and external environment. The $\mathrm{O}_{2}$ content in the soil in turn decreases, which leads 
to a reduction in respiration and reduces root growth [14]. Therefore, root zone hypoxic stress is further exacerbated by the use of plastic films.

The most effective means to cope with hypoxia has historically been to practise tillage. However, in recent years, various countermeasures and new techniques have emerged to address this issue, such as pump aeration [15], oxygenation [16], vacuum irrigation [17], micro/nano-bubble aeration (MNBA) [18] and the application of peroxides to the soil [19]. Injecting air into subsurface drip irrigation lines via an air compressor is an innovative approach referred to as pump aeration $[15,20]$. Oxygenation is the addition of $\mathrm{O}_{2}$ or hydrogen peroxide to drip irrigation or subsurface drip irrigation systems of crop root zones [16,19]. Vacuum irrigation has become a popular irrigation method in recent years, in which Venturi equipment is attached to the main irrigation line; this method uses a vacuum to introduce air into the irrigation system [21]. In addition, irrigation water can be treated with a micro/nano-bubble generator. This process results in irrigation water containing air bubbles with a diameter between that of microbubbles $(50 \mu \mathrm{m})$ and nanobubbles $(200 \mathrm{~nm})$, and the dissolved $\mathrm{O}_{2}$ concentration exceeds the $\mathrm{O}_{2}$ saturation value; this method is called micro/nanobubble irrigation (MNBA) [18]. These kinds of techniques can significantly improve the aerobic respiration of crop roots, increasing the soil microbial abundance and soil enzyme activity [17,22]. Many studies have also reported that soil aeration benefits crop yield and WUE. These techniques have been widely applied to herbs and orchards $[17,21,23]$. In response to these types of treatments, the yield of tomato (Solanum lycopersicum Mill.) [15], muskmelon [24], corn [17], cotton [25], onion [26], pepper [27], soybean, chickpea, pumpkin [26], and cucumber [28] have been shown to increase significantly, varying between $10 \%$ and $33 \%$. Moreover, the qualities of the crops also improved.

Tomato is one of the most widely grown vegetable crop species in the world, and greenhouse production of tomato has been rapidly increasing [29]. Tomato is a high-value crop species whose fruits are widely appreciated for their characteristic aroma and flavour, bright red colour, juicy texture, and sweetness, all of which have played a major role in its rapid and widespread adoption as an important food commodity worldwide. Numerous previous studies have investigated the effects of soil aeration and PFM on tomato [20,30]. To our knowledge, few studies have examined the combined effects of soil aeration and PFM on tomato growth, quality and yield $[15,21]$.

PFM and irrigation inhibit plant growth due to a lack of oxygen, and combined with one of the aeration treatments, are likely to diminish the negative effects of root zone oxygen stress. The objectives of this study were therefore to analyse the effects of MNBA and subsurface artificial air layer aeration (SAALA) on tomato dry matter accumulation, yield, irrigation water-use efficiency (IWUE), flavour, and nutrition and shape indices under three levels of PFM.

\section{Materials and Methods}

\subsection{Site Description, Soil Details and Test Materials}

This experiment was conducted in a greenhouse located at the $\mathrm{Xi}^{\prime}$ an Agricultural Technology Spread centre $\left(34^{\circ} 03^{\prime} \mathrm{N}, 108^{\circ} 52^{\prime} \mathrm{E}\right), \mathrm{Xi}^{\prime}$ an, Shaanxi Province, China, from March 2019 to January 2020. This site is on the Guanzhong Plain, which is a major food production area in northwestern China and is located at the southern edge of the Loess Plateau. The site is in the warm temperate, semihumid monsoon zone. The mean annual temperature is approximately $13.3^{\circ} \mathrm{C}$, the mean annual rainfall is approximately $552.2 \mathrm{~mm}$, the average sunshine duration is $2225 \mathrm{~h}$, and the mean annual frost-free period is 223 days. The greenhouse was $100 \mathrm{~m}$-long in the north-south direction and $12 \mathrm{~m}$-wide in the eastwest direction. Tomato (Solanum lycopersicum Mill. 'Jingfan 401') plants (Jing-Yan-Yi-Nong Seed Sci-tech Co., Ltd., Beijing, China) were transplanted and harvested on 27 March 2019 and on 25 July 2019 for spring cultivation and on 23 August 2019 and 30 January 2020 for autumn cultivation. The physical and chemical soil properties of the upper soil layer $(0-50 \mathrm{~cm})$ at the experimental site were measured. Clay $(<0.002 \mathrm{~mm}$ diameter soil 
particles) accounted for $29.9 \%$ of the upper soil layer, silt $(0.02-0.002 \mathrm{~mm}$ diameter soil particles) accounted for $43.9 \%$, and gravel (2-0.02 mm diameter soil particles) accounted for $26.2 \%$. The bulk density of the soil was $1.45 \mathrm{~g} \mathrm{~cm}^{-3}$, the field capacity was $27.12 \%$, the soil porosity was $49.12 \%$, and the $\mathrm{pH}$ was 7.65 . The soil consisted of $14.1 \mathrm{~g} \mathrm{~kg}^{-1}$ organic matter, $1.31 \mathrm{~g} \mathrm{~kg}^{-1}$ total nitrogen, $66.9 \mathrm{mg} \mathrm{kg}^{-1}$ available nitrogen, $9.52 \mathrm{~g} \mathrm{~kg}^{-1}$ total phosphorus, $94.0 \mathrm{mg} \mathrm{kg}^{-1}$ available phosphorus, $1.82 \mathrm{~g} \mathrm{~kg}^{-1}$ total potassium, and $89.1 \mathrm{mg} \mathrm{kg}^{-1}$ available potassium.

\subsection{Experimental Design and Treatments}

Each plot was $3.7 \mathrm{~m}$ in length and $1.5 \mathrm{~m}$ wide, with a total planting area of $5.55 \mathrm{~m}^{2}$. Two subsurface drip irrigation tubes (Qinchuan Water-saving Irrigation Equipment Engineering Co., Ltd., Yangling, China) with diameters of $16 \mathrm{~mm}$ were established in each cultivation area, and the spacing between the drip irrigation tubes was $0.50 \mathrm{~m}$. Twenty-five-day-old seedlings were transplanted into the plots. The detailed information is shown in Figure A1. Further, 18 tomato seedlings plot $^{-1}$ were planted in double rows, with a plant spacing of $0.40 \mathrm{~m}$ and row spacing of $0.50 \mathrm{~m}$ (Figure A1a). Guard rows were set up at both ends of the experimental field. The ridge width was $0.70 \mathrm{~m}$, the height was $0.15 \mathrm{~m}$, and the crop rows were aligned east to west (Figure A1a). The plastic film (Yinchuan Dingheng Plastic Film, Co., Ltd., Yinchuan, China) used was polyethylene (thickness, 0.026-0.029 mm; width, $1.20 \mathrm{~m}$; colourless; density, $0.92 \mathrm{~kg} \mathrm{dm}^{-3}$; tensile strength, 18-24 MPa).

The experimental design was a $3 \times 3$ full-factorial design. The experiment was arranged as a randomized complete block design, with three replicates for each combination of treatments, for a total of nine replicates ( 3 treatment repetitions $\times 3$ sample repetitions). The experimental arrangement details are shown in Table 1. The experiment involved 3 levels of PFM (treatments), with F, H and N representing full, half and no treatments, respectively (Figure A1b). Similarly, SAALA, MNBA and CK represent subsurface artificial air layer aeration, micro/nano-bubble water aeration and no aeration treatment, respectively.

Table 1. Experimental design for the 9 treatment combinations.

\begin{tabular}{ccc}
\hline Treatment Number & Plastic Film Mulching Treatment & Aeration Treatment \\
\hline 1 & F & CK \\
2 & H & CK \\
3 & N & SAALA \\
4 & F & SAALA \\
5 & H & SAALA \\
6 & MNBA \\
7 & F & MNBA \\
8 & H & MNBA \\
\hline
\end{tabular}

Note: F, full film covering; H, half film covering; N, no film covering; CK, no aeration treatment; SAALA, subsurface artificial air layer aeration; MNBA, micro-nano-bubble water aeration.

Figure 1a shows the schematic field layout of the SAALA system. The concentration of $\mathrm{O}_{2}$ in the atmosphere was higher than that in the soil. The rate of air exchange between the air and soil can be accelerated by SAALA. An air layer was dug before planting; the width of trench was $0.70 \mathrm{~m}$, the length was $3.70 \mathrm{~m}$, and the depth was $0.45 \mathrm{~m}$. An iron screen was laid at a depth of $15 \mathrm{~cm}$ at the bottom of the groove, and gauze was added to cover the iron screen. Then, a layer of soil (15 cm deep) was added on top of the iron screen. To increase the $\mathrm{O}_{2}$ concentration in the subsurface artificial air layer, both ends of the subsurface artificial air layer were connected to the outside air. Three plastic pipes were placed in the plots to connect the subsurface artificial air layer and outside air to increase the $\mathrm{O}_{2}$ concentration in the subsurface artificial air layer. As shown in Figure 1b, the MNBA system consisted of several parts, including a water storage tank, an immersible pump, a filter, and a micro/nano-bubble generator (BS P-70, Besson Co., Ltd., Jinan, China). The main technical parameters of the micro/nano-bubble generator are shown in Table A1. Four micro/nano-bubble generators were arranged at the bottom of the tank. Air was 
mixed with water in the tank by a micro/nano-bubble generator, and then high-density, uniform air was injected into the soil through the subsurface drip irrigation system. The $\mathrm{CK}$ treatment was irrigated exclusively with drip irrigation.

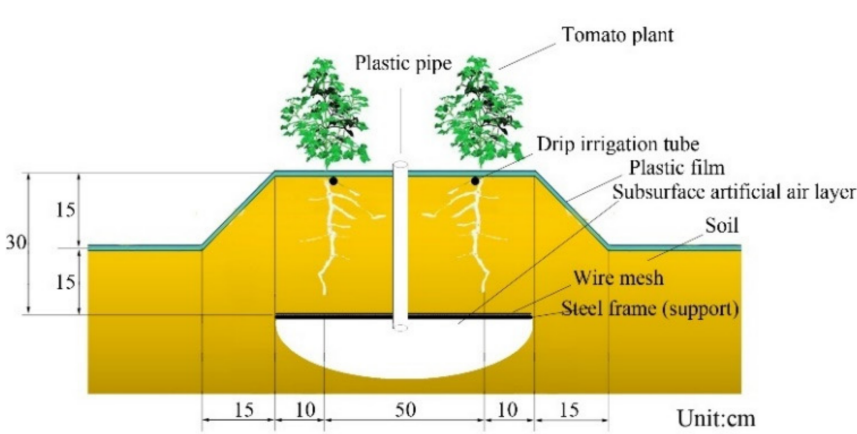

(a) Subsurface artificial air layer

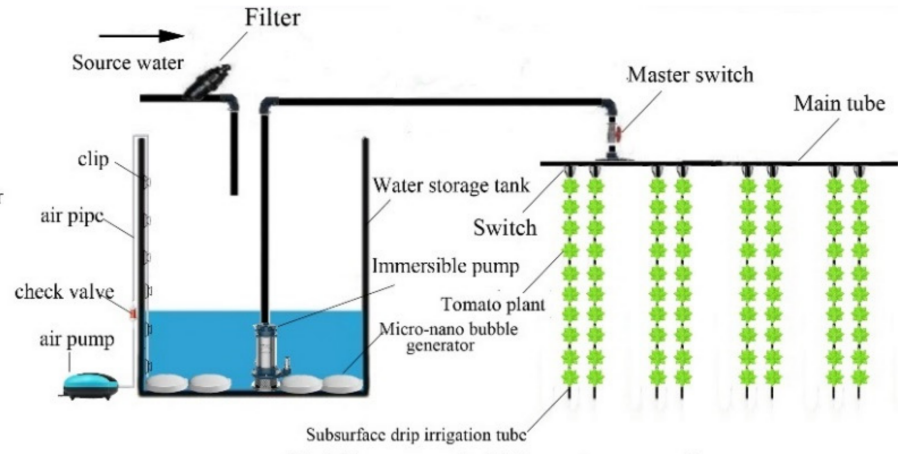

(b) Micro-nano bubble water oxygation

Figure 1. Sketch map of (a) subsurface artificial air layer aeration and (b) micro-nano-bubble water aeration.

Before transplanting, the soil was rototilled, and the experimental fields were fertilized with $300 \mathrm{~kg} \mathrm{ha}^{-1}$ of compound fertilizer $\left(18 \% \mathrm{~N}, 15 \% \mathrm{P}_{2} \mathrm{O}_{5}\right.$, and $\left.12 \% \mathrm{~K}_{2} \mathrm{O}\right)$ and $900 \mathrm{~kg} \mathrm{ha}^{-1}$ of diammonium phosphate $\left(18 \% \mathrm{~N}\right.$ and $\left.46 \% \mathrm{P}_{2} \mathrm{O}_{5}\right)$. The fertilizer was broadcast uniformly as a basal fertilizer in each plot. Plants were irrigated at 5-day intervals using the drip irrigation system. The irrigation amount was 1.2 times the water surface evaporation, with the same irrigation amount applied to each plot. Detailed information on the irrigation date and amount is specified in Table A2. During the experimental period, all agronomic management practices for tomato, including pollination, branch stem pruning, pest control, etc., were consistent with local production practices.

\subsection{Measurements}

\subsubsection{Fruit, Dry Matter Accumulation and IWUE}

Fruit yield was measured during the fruit mature period, and the spring and autumn tomato fruits were harvested at 100-120 and 130-160 days after transplanting, respectively. Dry matter accumulation (stem, leaf, and root dry matter) was measured after all fruit yields were measured. The IWUE, defined as the ratio of fruit yield $(\mathrm{kg})$ to the irrigation amount, was calculated as IWUE $=\left(\mathrm{Y} \mathrm{I}^{-1}\right)$, where IWUE is the irrigation water-use efficiency $\left(\mathrm{kg} \mathrm{m}^{-3}\right), \mathrm{Y}$ is the fruit yield $(\mathrm{kg})$ and $\mathrm{I}$ is the amount of applied water $\left(\mathrm{m}^{3}\right)$.

\subsubsection{Shape, Nutrition and Flavour of Tomato Fruits}

The shape, nutrition and flavour of fresh tomato fruits were measured. Fruit width and length of the first 9 ripe fruits were measured using a digital Vernier calliper (MNT150T, Mei-Nai-Te Co., Ltd., Shanghai, China). The hardness of the fruits was determined using a hardness tester (GY-1, Tuo-Pu-Yun-Nong Co., Ltd., HangZhou, China). After the measurement of fruit shape, the fruits were sliced and juiced with a domestic juicer after removing the skin and seeds. The juice was decanted and subjected to a series of tests to measure the following nutrition and flavour parameters: vitamin C (VC) content, soluble solids content, lycopene content, soluble protein content, soluble sugars and titratable acid. The VC content was measured by molybdenum blue colourimetry. This method is based on the reaction of ascorbic acid (VC) with ammonium phosphomolybdate in the presence of $\mathrm{SO}_{4}{ }^{2-}$ and $\mathrm{PO}_{4}{ }^{3-}$, generating blue molybdenum, which has a maximum absorption at $760 \mathrm{~nm}$. This VC assay method is accurate, repeatable, and insensitive to interference by the presence of common reducing sugars [31]. A hand-held refractometer (Atago Co., Ltd., Tokyo, Japan) was used to directly measure the soluble solids of the blended fruits. Lycopene was extracted with $2 \%$ dichloromethane and petroleum as solvents to enhance the solubility of lycopene, and the absorption at $502 \mathrm{~nm}$ was subsequently measured 
by an ultraviolet spectrophotometer [32]. The soluble protein content was determined using Coomassie brilliant blue [31], and the soluble sugar content was measured by the anthrone colourimetry method [31]. Titratable acid was determined by diluting an aliquot of the blended fruits and titrating against $0.1 \mathrm{~mol} \mathrm{~L}^{-1} \mathrm{NaOH}$ using phenolphthalein as an indicator [33]. Last, the sugar/acid ratio was determined by dividing the soluble sugar concentration by the titratable acid content.

\subsubsection{Economic Analysis and Evaluation}

Economic analysis and evaluation were based on additional labour, electricity and plastic film mulching cost, as well as the depreciation of equipment of each treatment compared with CK. The total income was calculated by multiplying the total yield by local average price for the year.

\subsection{Data Analysis}

Data from the experiment were analysed statistically using SPSS 22 (IBM, Armonk, New York, NY, USA). The experimental data were analysed using two-way ANOVA in SPSS. Multiple comparisons using Duncan's test were completed when the ANOVA indicated significant differences $(p \leq 0.05)$. All figures were constructed using the graphing software OriginPro 9.0 (Origin Lab Corporation, Northampton, MA, USA) and Photoshop CS5 (Adobe Systems, Inc., San Jose, CA, USA).

\section{Results}

\subsection{Effects of Soil Aeration and PFM on Dry Matter Accumulation of Tomato}

As shown in Table 2, both the MNBA and SAALA treatments had a significant effect on dry matter accumulation. Moreover, PFM increased the dry matter accumulation of tomato under each aeration treatment in both the spring and autumn seasons. The total dry weight of the tomato plants was 100.11-131.67 $\mathrm{g} \mathrm{plant}^{-1}$ in the spring and 80.36-120.23 $\mathrm{g} \mathrm{plant}^{-1}$ in autumn. During the spring season, PFM had a significant effect on the leaf, root, and total dry weight of the tomato plants but not on the stem dry weight or the root/shoot ratio. Soil aeration had a significant effect on the leaf, root, and total dry weight of the tomato plants. Compared with PFM, soil aeration resulted in significant increases in the stem, leaf, root, and total dry weight of tomato plants. During the autumn season, the results showed that PFM had a significant effect on the stems, leaves, roots, total dry weight and root/shoot ratio of the tomato plants. Furthermore, soil aeration had a significant effect on the root and total dry weight of the tomato plants but not on the stems, leaves or root/shoot ratio. The ANOVA F-value showed that the interaction between soil aeration and PFM was not significant for any of the variables.

Table 2. Effects of different aeration treatments on the stem, leaf and root dry weight and the root-shoot ratio of a single tomato for spring and autumn tomatoes 120 and 160 days after planting.

\begin{tabular}{|c|c|c|c|c|c|c|c|c|c|c|}
\hline \multirow{2}{*}{$\begin{array}{c}\text { Season } \\
\text { Treatments }\end{array}$} & \multicolumn{5}{|c|}{ Tomato in Spring } & \multicolumn{5}{|c|}{ Tomato in Autumn } \\
\hline & Stem (g) & Leaves/(g) & Root/(g) & $\begin{array}{c}\text { Total Dry } \\
\text { Weight/(g) }\end{array}$ & $\begin{array}{l}\text { Root-Shoot } \\
\text { Ratio/(\%) }\end{array}$ & Stems (g) & Leaves/(g) & Root/(g) & $\begin{array}{c}\text { Total Dry } \\
\text { Weight/(g) }\end{array}$ & $\begin{array}{l}\text { Root-Shoot } \\
\text { Ratio/(\%) }\end{array}$ \\
\hline F-CK & $58.0 \pm 19.9 \mathrm{ab}$ & $48.9 \pm 10.2 \mathrm{bc}$ & $6.6 \pm 0.4 \mathrm{bc}$ & $113.5 \pm 22.8 \mathrm{bc}$ & $0.07 \pm 0.02 \mathrm{ab}$ & $56.8 \pm 12.0 \mathrm{ab}$ & $40.5 \pm 7.0 \mathrm{bc}$ & $5.2 \pm 0.4 \mathrm{bcd}$ & $102.5 \pm 9.5 \mathrm{bc}$ & $0.05 \pm 0.01 \mathrm{~d}$ \\
\hline $\mathrm{H}-\mathrm{CK}$ & $55.3 \pm 1.6 \mathrm{ab}$ & $46.0 \pm 8.2 \mathrm{bc}$ & $6.3 \pm 1.9 \mathrm{bc}$ & $107.5 \pm 7.3 \mathrm{bc}$ & $0.06 \pm 0.02 \mathrm{ab}$ & $47.6 \pm 16.5 \mathrm{bc}$ & $42.9 \pm 7.7 \mathrm{ab}$ & $4.4 \pm 0.9 \mathrm{~d}$ & $94.9 \pm 21.5 \mathrm{bcd}$ & $0.05 \pm 0.01 \mathrm{~cd}$ \\
\hline N-CK & $51.9 \pm 2.8 \mathrm{~b}$ & $42.8 \pm 2.1 \mathrm{c}$ & $5.5 \pm 0.3 \mathrm{c}$ & $100.1 \pm 2.1 \mathrm{c}$ & $0.06 \pm 0 \mathrm{~b}$ & $41.9 \pm 8.4 \mathrm{c}$ & $33.8 \pm 7.3 \mathrm{a}$ & $4.7 \pm 0.2 \mathrm{~d}$ & $80.4 \pm 15.5 \mathrm{~d}$ & $0.08 \pm 0.02 \mathrm{ab}$ \\
\hline F-SAALA & $65.8 \pm 0.0 \mathrm{a}$ & $57.4 \pm 1.6 \mathrm{a}$ & $8.5 \pm 1.5 \mathrm{a}$ & $131.7 \pm 1.7 \mathrm{a}$ & $0.07 \pm 0.01 \mathrm{ab}$ & $63.5 \pm 7.9 \mathrm{a}$ & $49.4 \pm 6.1 \mathrm{a}$ & $7.3 \pm 0.2 \mathrm{a}$ & $120.2 \pm 10.7 \mathrm{a}$ & $0.05 \pm 0 \mathrm{~d}$ \\
\hline H-SAALA & $62.3 \pm 15.6 \mathrm{ab}$ & $49.2 \pm 10.4 \mathrm{abc}$ & $7.0 \pm 0.4 \mathrm{~b}$ & $118.5 \pm 18.5 \mathrm{ab}$ & $0.06 \pm 0.01 \mathrm{ab}$ & $50.7 \pm 13.2 \mathrm{bc}$ & $43.4 \pm 11.4 \mathrm{ab}$ & $7.1 \pm 0.8 \mathrm{a}$ & $101.1 \pm 20.5 \mathrm{bc}$ & $0.07 \pm 0.01 \mathrm{bc}$ \\
\hline $\begin{array}{l}\text { N-SAALA } \\
\text { F-MNBAA }\end{array}$ & $\begin{array}{l}59.6 \pm 1.6 \mathrm{ab} \\
609 \pm 130 \mathrm{a}\end{array}$ & $\begin{array}{l}46.6 \pm 8.0 \mathrm{bc} \\
5183+888 \mathrm{a}\end{array}$ & $6.6 \pm 0.6 \mathrm{bc}$ & $112.8 \pm 8.6 \mathrm{bc}$ & $0.06 \pm 0.01 \mathrm{ab}$ & $50.5 \pm 11.5 \mathrm{bc}$ & $35.7 \pm 6.1 \mathrm{bc}$ & $5.5 \pm 0.3 \mathrm{bc}$ & $91.8 \pm 17.3 \mathrm{bcd}$ & $0.09 \pm 0.01 \mathrm{a}$ \\
\hline $\begin{array}{l}\text { F-MNBAA } \\
\text { H-MNBA }\end{array}$ & $\begin{array}{l}60.5 \pm 4.8 \mathrm{ab} \\
60.5 \pm 13.0 \mathrm{~b}\end{array}$ & $\begin{array}{l}511.83 \pm 8.8 \mathrm{ab} \\
48.47 \pm 8.7 \mathrm{bc}\end{array}$ & $\begin{array}{l}8.4 \pm 1.5 \mathrm{a} \\
7.2 \pm 1.2 \mathrm{~b}\end{array}$ & $\begin{array}{c}121.2 \pm 14.9 \mathrm{ab} \\
116.1 \pm 8.5 \mathrm{~b}\end{array}$ & $\begin{array}{l}0.08 \pm 0.02 \mathrm{a} \\
0.07 \pm 0.01 \mathrm{ab}\end{array}$ & $\begin{array}{l}57.5 \pm 10.7 \mathrm{zab} \\
50.5 \pm 10.4 \mathrm{bc}\end{array}$ & $\begin{array}{l}449.6 \pm 10.8 \mathrm{ab} \\
39.6 \pm 8 \mathrm{bc}\end{array}$ & $\begin{array}{l}5.9 \pm 0.3 \mathrm{~b} \\
5.0 \pm 1.6 \mathrm{~cd}\end{array}$ & $\begin{array}{l}108.1 \pm 10.1 \mathrm{ab} \\
95.1 \pm 18 \mathrm{bcd}\end{array}$ & $\begin{array}{l}0.07 \pm 0.01 \mathrm{~b} \\
0.05 \pm 0.01 \mathrm{~cd}\end{array}$ \\
\hline N-MNBA & $52.4 \pm 9.9 \mathrm{~b}$ & $46.94 \pm 6.6 \mathrm{bc}$ & $7.3 \pm 0.3 \mathrm{~b}$ & $106.7 \pm 13.0 \mathrm{bc}$ & $0.08 \pm 0.01 \mathrm{a}$ & $41.7 \pm 4.7 \mathrm{c}$ & $39.4 \pm 5.0 \mathrm{bc}$ & $4.4 \pm 0.9 \mathrm{~d}$ & $85.7 \pm 9.1 \mathrm{~cd}$ & $0.06 \pm 0.02 \mathrm{bc}$ \\
\hline $\begin{array}{l}\text { Plastic film } \\
\text { film }\end{array}$ & $2.4 \mathrm{~ns}$ & 4.4 * & $8.0 * *$ & $7.4^{* *}$ & $1.0 \mathrm{~ns}$ & $9.6 * *$ & 6.1 ** & $6.3 * *$ & 12.5 ** & 13.6 ** \\
\hline $\begin{array}{l}\text { mulccung (I) } \\
\text { Aeration (A) }\end{array}$ & $4.1^{*}$ & 4.7 * & $26.5 * *$ & $11.3^{* *}$ & $3.5 \mathrm{~ns}$ & $1.7 \mathrm{~ns}$ & $2.2 \mathrm{~ns}$ & $21.2 * *$ & $4.0 *$ & $1.3 \mathrm{~ns}$ \\
\hline $\mathrm{P} \times \mathrm{A}$ & $0.1 \mathrm{~ns}$ & $0.2 \mathrm{~ns}$ & $1.4 \mathrm{~ns}$ & $0.1 \mathrm{~ns}$ & $0.5 \mathrm{~ns}$ & $0.0 \mathrm{~ns}$ & $1.4 \mathrm{~ns}$ & $2.8 \mathrm{~ns}$ & $0.4 \mathrm{~ns}$ & $1.0 \mathrm{~ns}$ \\
\hline
\end{tabular}

Note: $\mathrm{F}$, full film covering; $\mathrm{H}$, half film covering; $\mathrm{N}$, no film covering; $\mathrm{CK}$, no aeration treatment; SAALA subsurface artificial air layer aeration; MNBA, micro-nano-bubble water aeration. Data are presented as the mean \pm standard deviation $(n=9)$. Different lowercase letters in the same column indicate significant differences between treatments (Duncan's test, $p<0.05$ ). ANOVA F-values for main and interaction effects were not significant (ns) or were significant at the $\leq 5 \%\left(^{*}\right)$ and $\leq 1 \%\left({ }^{* *}\right)$ levels. Bold number indicate significant differences at the $\leq 5 \%$ level. 


\subsection{Effects of Soil Aeration and PFM on Fruit Yield and IWUE}

The effects of soil aeration and PFM on the fruit yield and IWUE of tomato are shown in Table 3. The fruit yield and IWUE in the spring season were higher than those in the autumn season. Fruit yield and IWUE increased with increasing PFM in response to the $\mathrm{N}, \mathrm{H}$ to $\mathrm{F}$ treatments during both the spring and autumn seasons. In the spring, the $\mathrm{F}$ treatment under the CK, SAALA and MNBA treatments increased the fruit yield by $35.5 \%$, $31.1 \%$, and $29.2 \%$, respectively, compared with the yield of the $\mathrm{N}$ treatment. Both the MNBA and SAALA treatments had a significantly higher fruit yield and IWUE than did the CK treatment during both the spring and autumn seasons. PFM had a significant $(p \leq 0.01)$ impact on fruit yield and IWUE in the spring season but no significant impact during autumn. The interaction between aeration and PFM had no significant $(p>0.05)$ effect on fruit yield or IWUE.

Table 3. Effects of different aeration treatments on fruit yield and irrigation water-use efficiency (IWUE) of tomato.

\begin{tabular}{|c|c|c|c|c|}
\hline \multirow{2}{*}{$\begin{array}{c}\text { Season } \\
\text { Treatments }\end{array}$} & \multicolumn{2}{|c|}{ Tomato in Spring } & \multicolumn{2}{|c|}{ Tomato in Autumn } \\
\hline & Fruit Yield (g/plant) & $\begin{array}{l}\text { IWUE for Fruit } \\
\left(\mathrm{kg} / \mathrm{m}^{3}\right)\end{array}$ & Fruit Yield (g/plant) & $\begin{array}{l}\text { IWUE for Fruit } \\
\left(\mathrm{kg} / \mathrm{m}^{3}\right)\end{array}$ \\
\hline $\mathrm{F}-\mathrm{CK}$ & $2412.9 \pm 616.9 \mathrm{abc}$ & $21.7 \pm 5.6 \mathrm{abc}$ & $1995.6 \pm 471.4 \mathrm{bc}$ & $14.1 \pm 3.3 \mathrm{bc}$ \\
\hline $\mathrm{H}-\mathrm{CK}$ & $2068.8 \pm 730.3 \mathrm{bc}$ & $18.6 \pm 6.6 \mathrm{bc}$ & $1904.4 \pm 388.8 \mathrm{bc}$ & $13.5 \pm 2.8 \mathrm{bc}$ \\
\hline N-CK & $1781.3 \pm 174.7 \mathrm{c}$ & $16.0 \pm 1.6 \mathrm{c}$ & $1737.8 \pm 330.4 \mathrm{c}$ & $12.3 \pm 2.3 c$ \\
\hline F-SAALA & $3014.0 \pm 580.3 \mathrm{a}$ & $27.1 \pm 5.2 \mathrm{a}$ & $2512.2 \pm 79.7 \mathrm{a}$ & $17.8 \pm 0.6 \mathrm{a}$ \\
\hline H-SAALA & $2519.2 \pm 585.5 \mathrm{ab}$ & $22.7 \pm 5.3 \mathrm{ab}$ & $2090.0 \pm 370.05 \mathrm{ab}$ & $14.8 \pm 2.6 \mathrm{bc}$ \\
\hline N-SAALA & $2299.4 \pm 318.5 \mathrm{bc}$ & $20.7 \pm 2.9 \mathrm{bc}$ & $2136.7 \pm 290.3 \mathrm{abc}$ & $15.1 \pm 2.1 \mathrm{abc}$ \\
\hline F-MNBA & $2602.4 \pm 604.1 \mathrm{ab}$ & $23.4 \pm 5.4 \mathrm{ab}$ & $2166.7 \pm 333.3 \mathrm{ab}$ & $15.3 \pm 2.4 \mathrm{ab}$ \\
\hline H-MNBA & $2210.3 \pm 419.2 \mathrm{bc}$ & $19.9 \pm 3.8 \mathrm{bc}$ & $2204.4 \pm 484.9 \mathrm{ab}$ & $15.6 \pm 3.4 \mathrm{ab}$ \\
\hline N-MNBA & $2014.8 \pm 767.9 \mathrm{bc}$ & $\begin{array}{l}18.1 \pm 6.9 \mathrm{bc} \\
\text { F-value }\end{array}$ & $2081.1 \pm 402.1 \mathrm{bc}$ & $14.7 \pm 2.9 \mathrm{bc}$ \\
\hline Plastic film mulching $(\mathrm{P})$ & $6.9 * *$ & $6.9 * *$ & $2.3 \mathrm{~ns}$ & $2.3 \mathrm{~ns}$ \\
\hline Aeration $(\mathrm{A})$ & $6.3 *$ & $6.3^{*}$ & $11.9^{* *}$ & $11.9 * *$ \\
\hline $\mathrm{P} \times \mathrm{A}$ & $0.0 \mathrm{~ns}$ & $0.0 \mathrm{~ns}$ & $0.2 \mathrm{~ns}$ & $0.2 \mathrm{~ns}$ \\
\hline
\end{tabular}

Note: F, full film covering; $\mathrm{H}$, half film covering; $\mathrm{N}$, no film covering; $\mathrm{CK}$, no aeration treatment; SAALA, subsurface artificial air layer aeration; MNBA, micro-nano-bubble water aeration. Data are presented as the mean \pm standard deviation $(n=9)$. Different lowercase letters in the same column indicate significant differences between treatments (Duncan's test, $p<0.05$ ). ANOVA F-values for main and interaction effects were not significant (ns) or were significant at the $\leq 5 \%\left(^{*}\right)$ and $\leq 1 \%\left({ }^{* *}\right)$ levels. Bold number indicate significant differences at the $\leq 5 \%$ level.

\subsection{Effects of Soil Aeration and PFM on Tomato Flavour Indices}

The data presented in Table 4 show that increasing the mulch-covered area significantly and progressively decreased the soluble sugar and titratable acid contents in both the spring and autumn seasons. The soluble sugar content decreased from $3.61 \%$ to $2.99 \%$ when the mulch-covered area changed from the $\mathrm{N}$ to $\mathrm{F}$ levels during the spring season and decreased from $5.11 \%$ to $3.61 \%$ during autumn. A similar situation of PFM-induced decreases in flavour indices also occurred under the MNBA and SAALA treatments. Soil aeration, mulch-covered area and the aeration volume $\times$ line depth interaction had significant effects on the sugar/acid ratio during the autumn season but had no significant effects during spring. Moreover, aeration had a significant impact on the titratable acid content during spring and had a significant impact on the soluble sugar content and sugar/acid ratio during autumn. The two-factor interaction had a significant impact on the titratable acid content during the spring season and on the sugar/acid ratio during the autumn season. 
Table 4. Effects of different aeration treatments on flavour indices of tomato.

\begin{tabular}{|c|c|c|c|c|c|c|}
\hline \multirow{2}{*}{ Treatments } & \multicolumn{3}{|c|}{ Tomato in Spring } & \multicolumn{3}{|c|}{ Tomato in Autumn } \\
\hline & $\underset{(\%)}{\text { Soluble Sugar }}$ & $\begin{array}{l}\text { Titratable Acid } \\
\text { (Weight \%) }\end{array}$ & $\begin{array}{c}\text { Sugar/Acid } \\
\text { Ratio }\end{array}$ & $\begin{array}{c}\text { Soluble Sugar } \\
(\%)\end{array}$ & $\begin{array}{l}\text { Titratable Acid } \\
\text { (Weight \%) }\end{array}$ & $\begin{array}{l}\text { Sugar/Acid } \\
\text { Ratio }\end{array}$ \\
\hline F-CK & $3.0 \pm 0.6 \mathrm{~b}$ & $0.41 \pm 0.02 \mathrm{~d}$ & $7.2 \pm 1.3 \mathrm{ab}$ & $3.6 \pm 0.4 \mathrm{bc}$ & $0.95 \pm 0.04 c$ & $3.8 \pm 0.5 \mathrm{~b}$ \\
\hline $\mathrm{H}-\mathrm{CK}$ & $3.5 \pm 1.2 \mathrm{ab}$ & $0.46 \pm 0.03 c$ & $7.6 \pm 2.9 \mathrm{ab}$ & $3.5 \pm 1.2 \mathrm{bc}$ & $1.05 \pm 0.06 \mathrm{ab}$ & $3.3 \pm 1.2 b$ \\
\hline N-CK & $3.6 \pm 0.4 \mathrm{ab}$ & $0.47 \pm 0.02 \mathrm{abc}$ & $7.7 \pm 0.8 \mathrm{ab}$ & $5.1 \pm 1.5 \mathrm{a}$ & $0.97 \pm 0.09 \mathrm{bc}$ & $5.4 \pm 1.7 \mathrm{a}$ \\
\hline F-SAALA & $3.6 \pm 0.2 \mathrm{ab}$ & $0.47 \pm 0.01 \mathrm{abc}$ & $7.8 \pm 0.4 \mathrm{ab}$ & $3.4 \pm 1.0 \mathrm{bc}$ & $0.94 \pm 0.01 \mathrm{c}$ & $3.6 \pm 1.1 \mathrm{~b}$ \\
\hline H-SAALA & $3.2 \pm 1.0 \mathrm{ab}$ & $0.47 \pm 0 \mathrm{abc}$ & $7.2 \pm 2.2 \mathrm{ab}$ & $3.6 \pm 0.2 \mathrm{bc}$ & $1 \pm 0.07 \mathrm{abc}$ & $3.6 \pm 0.3 \mathrm{~b}$ \\
\hline N-SAALA & $4.1 \pm 0.6 \mathrm{a}$ & $0.48 \pm 0.02 \mathrm{a}$ & $8.7 \pm 1.4 \mathrm{a}$ & $4.2 \pm 0.6 \mathrm{~b}$ & $1.07 \pm 0.08 \mathrm{a}$ & $3.9 \pm 0.8 \mathrm{~b}$ \\
\hline F-MNBA & $3.0 \pm 0.8 \mathrm{~b}$ & $0.46 \pm 0.02 \mathrm{bc}$ & $6.6 \pm 1.7 \mathrm{~b}$ & $3.1 \pm 0.7 c$ & $0.99 \pm 0.1 \mathrm{abc}$ & $3.1 \pm 0.8 \mathrm{~b}$ \\
\hline H-MNBA & $3.4 \pm 0.5 \mathrm{ab}$ & $0.47 \pm 0.03 \mathrm{abc}$ & $7.2 \pm 1.1 \mathrm{ab}$ & $3.4 \pm 0.5 \mathrm{bc}$ & $1.02 \pm 0.12 \mathrm{abc}$ & $3.4 \pm 0.5 \mathrm{~b}$ \\
\hline N-MNBA & $4.0 \pm 0.3 \mathrm{a}$ & $0.48 \pm 0.01 \mathrm{ab}$ & $\begin{array}{c}8.3 \pm 0.7 \mathrm{ab} \\
\text { F-value }\end{array}$ & $4.0 \pm 0.3 \mathrm{~b}$ & $1.01 \pm 0.07 \mathrm{abc}$ & $4.0 \pm 0.4 \mathrm{~b}$ \\
\hline $\begin{array}{l}\text { Plastic film } \\
\text { mulching }(\mathrm{P})\end{array}$ & $4.9 * *$ & $20.8 * *$ & $1.9 \mathrm{~ns}$ & $14.1^{* *}$ & $5.3 * *$ & $12.0 * *$ \\
\hline Aeration (A) & $1.7 \mathrm{~ns}$ & $33.2 * *$ & $0.0 \mathrm{~ns}$ & $4.8 *$ & $0.8 \mathrm{~ns}$ & $6.2 *$ \\
\hline $\mathrm{P} \times \mathrm{A}$ & $0.8 \mathrm{~ns}$ & $7.0 * *$ & $0.8 \mathrm{~ns}$ & $2.4 \mathrm{~ns}$ & $2.6 \mathrm{~ns}$ & $4.1 *$ \\
\hline
\end{tabular}

Note: F, full film covering; $\mathrm{H}$, half film covering; $\mathrm{N}$, no film covering; $\mathrm{CK}$, no aeration treatment; SAALA, subsurface artificial air layer aeration; MNBA, micro-nano-bubble water aeration. Data are presented as the mean \pm standard deviation $(n=9)$. Different lowercase letters in the same column indicate significant differences between treatments (Duncan's test, $p<0.05$ ). ANOVA F-values for main and interaction effects were not significant (ns) or were significant at the $\leq 5 \%\left(^{*}\right)$ and $\leq 1 \%\left({ }^{* *}\right)$ levels. Bold number indicate significant differences at the $\leq 5 \%$ level.

\subsection{Effects of Soil Aeration and PFM on Tomato Nutritional Quality}

The VC, soluble solids, lycopene and soluble protein contents were selected to characterize tomato nutritional quality and are presented in Table 5. Among the results of the single-factor analyses, PFM had a significant impact on the soluble solids, lycopene and soluble protein contents during spring and on the soluble protein content during autumn. In addition, the soluble solids and soluble protein contents gradually increased with increasing mulch-covered area. Fruit nutritional quality was found to be greater in the aeration treatments than in the CK treatment. Aeration had a significant impact on all four nutrition indices during spring and on the lycopene content and soluble protein content during autumn. However, there were no significant differences in soluble solids during autumn. The two-factor interaction had a significant impact on the lycopene and soluble protein contents during the spring season and on the VC content during the autumn season, but there was no significant impact on VC and soluble solids content during the spring season or on the soluble solids, lycopene, soluble protein contents during the autumn season.

Table 5. Effects of different aeration treatments on nutritional indices of tomato.

\begin{tabular}{|c|c|c|c|c|c|c|c|c|}
\hline \multirow{2}{*}{$\begin{array}{c}\text { Season } \\
\text { Treatments }\end{array}$} & \multicolumn{4}{|c|}{ Tomato in Spring } & \multicolumn{4}{|c|}{ Tomato in Autumn } \\
\hline & $\begin{array}{l}\text { Vitamin C } \\
\text { Content } \\
(\mathrm{mg} / 100 \mathrm{~g})\end{array}$ & $\begin{array}{c}\text { Soluble } \\
\text { Solids (\%) }\end{array}$ & $\begin{array}{c}\text { Lycopene } \\
\text { Content (ug/g) }\end{array}$ & $\begin{array}{c}\text { Soluble } \\
\text { Protein (\%) }\end{array}$ & $\begin{array}{c}\text { Vitamin C } \\
\text { Content } \\
(\mathrm{mg} / 100 \mathrm{~g})\end{array}$ & $\begin{array}{c}\text { Soluble } \\
\text { Solids (\%) }\end{array}$ & $\begin{array}{c}\text { Lycopene } \\
\text { Content (ug/g) }\end{array}$ & $\begin{array}{c}\text { Soluble } \\
\text { Protein (\%) }\end{array}$ \\
\hline F-CK & $21.5 \pm 0.9 c$ & $5.0 \pm 0.3 \mathrm{ab}$ & $51.3 \pm 5.5 b$ & $1.97 \pm 0.08 b c$ & $23.3 \pm 1.1 \mathrm{~b}$ & $4.9 \pm 0.4 \mathrm{a}$ & $42.9 \pm 4.5 \mathrm{~b}$ & $1.98 \pm 0.07 b$ \\
\hline $\mathrm{H}-\mathrm{CK}$ & $22.6 \pm 1.9 \mathrm{bc}$ & $4.9 \pm 0.3 \mathrm{bc}$ & $55.0 \pm 4.0 \mathrm{ab}$ & $1.96 \pm 0.08 \mathrm{bc}$ & $25.0 \pm 2.0 \mathrm{ab}$ & $4.9 \pm 0.2 \mathrm{a}$ & $41.8 \pm 5.3 \mathrm{~b}$ & $1.96 \pm 0.07 \mathrm{~b}$ \\
\hline N-CK & $23.6 \pm 1.4 \mathrm{bc}$ & $4.5 \pm 0.6 \mathrm{c}$ & $47.3 \pm 6.2 \mathrm{c}$ & $1.93 \pm 0.07 c$ & $25.9 \pm 2.6 \mathrm{a}$ & $4.7 \pm 0.7 \mathrm{a}$ & $43.6 \pm 2.0 \mathrm{~b}$ & $1.83 \pm 0.1 \mathrm{~b}$ \\
\hline F-SAALA & $25.3 \pm 3.1 \mathrm{ab}$ & $5.2 \pm 0.4 \mathrm{a}$ & $56.0 \pm 3.5 \mathrm{a}$ & $2.44 \pm 0.11 \mathrm{a}$ & $26.0 \pm 1.4 \mathrm{a}$ & $5.1 \pm 0.5 \mathrm{a}$ & $48.5 \pm 1.1 \mathrm{a}$ & $2.03 \pm 0.05 \mathrm{a}$ \\
\hline H-SAALA & $23.3 \pm 1.7 \mathrm{bc}$ & $5.1 \pm 0.5 \mathrm{ab}$ & $56.7 \pm 3.5 \mathrm{a}$ & $2.34 \pm 0.11 \mathrm{a}$ & $25.5 \pm 1.7 \mathrm{a}$ & $5.0 \pm 0.5 \mathrm{a}$ & $43.2 \pm 4.5 \mathrm{~b}$ & $1.99 \pm 0.09 \mathrm{~b}$ \\
\hline N-SAALA & $26.8 \pm 3.1 \mathrm{a}$ & $4.7 \pm 0.4 \mathrm{bc}$ & $56.3 \pm 2.9 \mathrm{a}$ & $1.95 \pm 0.1 \mathrm{bc}$ & $25.0 \pm 1.4 \mathrm{ab}$ & $4.9 \pm 0.5 \mathrm{a}$ & $43.2 \pm 2.0 \mathrm{~b}$ & $1.98 \pm 0.06 \mathrm{~b}$ \\
\hline F-MNBA & $25.0 \pm 2.7 \mathrm{ab}$ & $5.1 \pm 0.4 \mathrm{ab}$ & $55.7 \pm 2.9 \mathrm{a}$ & $2.38 \pm 0.1 \mathrm{a}$ & $25.6 \pm 1.3 \mathrm{a}$ & $5.1 \pm 0.3 \mathrm{a}$ & $45.2 \pm 4.6 \mathrm{ab}$ & $1.98 \pm 0.08 \mathrm{ab}$ \\
\hline H-MNBA & $24.6 \pm 3.3 \mathrm{ab}$ & $5.1 \pm 0.3 \mathrm{ab}$ & $56.0 \pm 2.5 \mathrm{a}$ & $2.39 \pm 0.11 \mathrm{a}$ & $25.4 \pm 1.9 \mathrm{a}$ & $4.8 \pm 0.2 \mathrm{a}$ & $43.9 \pm 1.4 b$ & $1.98 \pm 0.09 \mathrm{~b}$ \\
\hline N-MNBA & $25.0 \pm 2.7 \mathrm{ab}$ & $4.8 \pm 0.5 \mathrm{bc}$ & $55.1 \pm 2.6 \mathrm{ab}$ & $\begin{array}{c}2.03 \pm 0.07 \mathrm{~b} \\
\text { F-value }\end{array}$ & $25.1 \pm 2.5 \mathrm{ab}$ & $5.0 \pm 0.6 \mathrm{a}$ & $44.0 \pm 2.4 \mathrm{~b}$ & $1.92 \pm 0.08 b$ \\
\hline $\begin{array}{l}\text { Plastic film } \\
\text { mulching }(\mathrm{P})\end{array}$ & $2.6 \mathrm{~ns}$ & $7.5 * *$ & $6.0 * *$ & $36.4^{* *}$ & $2.4 \mathrm{~ns}$ & $1.0 \mathrm{~ns}$ & $2.2 \mathrm{~ns}$ & $10.5^{* *}$ \\
\hline $\begin{array}{c}\text { Aeration (A) } \\
\mathrm{P} \times \mathrm{A}\end{array}$ & $\begin{array}{l}\mathbf{1 6 . 2}{ }^{* *} \\
1.2 \mathrm{~ns}\end{array}$ & $\begin{array}{l}5.4^{*} \\
0.0 \mathrm{~ns}\end{array}$ & $\begin{array}{c}24.5^{* *} \\
4.6^{*}\end{array}$ & $\begin{array}{l}162.7^{* *} \\
25.3^{* *}\end{array}$ & $\begin{array}{l}1.6 \mathrm{~ns} \\
4.8^{*}\end{array}$ & $\begin{array}{l}1.6 \mathrm{~ns} \\
0.5 \mathrm{~ns}\end{array}$ & $\begin{array}{l}4.9^{*} \\
1.7 \mathrm{~ns}\end{array}$ & $\begin{array}{l}8.7 * * \\
2.9 \mathrm{~ns}\end{array}$ \\
\hline
\end{tabular}

Note: $\mathrm{F}$, full film covering; $\mathrm{H}$, half film covering; $\mathrm{N}$, no film covering; $\mathrm{CK}$, no aeration treatment; SAALA subsurface artificial air layer aeration; MNBA, micro-nano-bubble water aeration. Data are presented as the mean \pm standard deviation $(n=9)$. Different lowercase letters in the same column indicate significant differences between treatments (Duncan's test, $p<0.05$ ). ANOVA F-values for main and interaction effects were not significant (ns) or were significant at the $\leq 5 \%\left({ }^{*}\right)$ and $\leq 1 \%\left({ }^{* *}\right)$ levels. Bold number indicate significant differences at the $\leq 5 \%$ level. 


\subsection{Effects of Soil Aeration and PFM on Tomato Shape}

The impacts of soil aeration and PFM on the shape indices of tomato are shown in Table 6. During the spring season, PFM had a marked effect on fruit length, width, weight and hardness, and soil aeration markedly influenced the fruit width, length and individual weight. Aeration did not significantly affect the hardness in either the spring or autumn. Compared with the $\mathrm{N}$ treatment, PFM resulted in a higher fruit hardness. The $\mathrm{F}$ treatment presented had the highest fruit length, fruit width and individual fruit weight during spring. The interaction of soil aeration and PFM notably affected the fruit width. However, there were no significant differences in fruit length, width, and single fruit weight during autumn. Figure 2 shows the relationships between fruit weight, fruit width and fruit length. Figure $2 \mathrm{a}, \mathrm{b}$ shows the fruit width and fruit length are important factors that affect fruit shape, which is important for fruit weight. Figure 2c shows a high coefficient of determination for the relationship of tomato fruit width and fruit length during the aeration and PFM treatments. Relationships between fruit weight and width, fruit weight and length and fruit width and length were modelled using $\operatorname{In}(\mathrm{y})=2.64+0.31 \times \operatorname{In}(\mathrm{x})$, $\operatorname{In}(\mathrm{y})=2.28+0.36 \times \operatorname{In}(\mathrm{x})$ and $\operatorname{In}(\mathrm{y})=1.11+0.75 \times \operatorname{In}(\mathrm{x})$, respectively.

Table 6. Effects of different aeration treatments on shape indices of tomato.

\begin{tabular}{|c|c|c|c|c|c|c|c|c|}
\hline \multirow{2}{*}{$\begin{array}{c}\text { Season } \\
\text { Treatments }\end{array}$} & \multicolumn{4}{|c|}{ Tomato in Spring } & \multicolumn{4}{|c|}{ Tomato in Autumn } \\
\hline & $\begin{array}{l}\text { Fruit Length } \\
(\mathrm{mm})\end{array}$ & $\begin{array}{c}\text { Fruit Width } \\
(\mathrm{mm})\end{array}$ & $\begin{array}{l}\text { Single Fruit } \\
\text { Weight (g) }\end{array}$ & $\begin{array}{l}\text { Hardness } \\
\left(\mathrm{Kpa} / \mathrm{m}^{2}\right)\end{array}$ & $\begin{array}{l}\text { Fruit Length } \\
(\mathrm{mm})\end{array}$ & $\begin{array}{c}\text { Fruit Width } \\
(\mathrm{mm})\end{array}$ & $\begin{array}{l}\text { Single Fruit } \\
\text { Weight (g) }\end{array}$ & $\begin{array}{l}\text { Hardness } \\
\left(\mathrm{Kpa} / \mathrm{m}^{2}\right)\end{array}$ \\
\hline F-CK & $50.9 \pm 6.0 \mathrm{abc}$ & $54.7 \pm 1.4 \mathrm{ab}$ & $89.2 \pm 15.7 \mathrm{abc}$ & $10.6 \pm 0.8 \mathrm{~b}$ & $41.7 \pm 7.9 \mathrm{a}$ & $47.4 \pm 7.9 \mathrm{a}$ & $77.2 \pm 13.7 \mathrm{a}$ & $11.6 \pm 0.8 \mathrm{ab}$ \\
\hline $\mathrm{H}-\mathrm{CK}$ & $44.2 \pm 9.1 \mathrm{c}$ & $48.1 \pm 5.8 \mathrm{c}$ & $60.6 \pm 21.5 \mathrm{~d}$ & $11.4 \pm 0.9 \mathrm{ab}$ & $38.0 \pm 5.0 \mathrm{a}$ & $46.9 \pm 7.8 \mathrm{a}$ & $79.2 \pm 18.4 \mathrm{a}$ & $11.9 \pm 1.2 \mathrm{ab}$ \\
\hline N-CK & $44.1 \pm 4.6 \mathrm{c}$ & $51.7 \pm 3.8 \mathrm{bc}$ & $66.0 \pm 14.9 \mathrm{~cd}$ & $12.2 \pm 0.9 \mathrm{a}$ & $39.0 \pm 8.4 \mathrm{a}$ & $44.9 \pm 7.1 \mathrm{a}$ & $65.2 \pm 10.3 \mathrm{a}$ & $12.0 \pm 1.1 \mathrm{ab}$ \\
\hline F-SAALA & $54.3 \pm 6.7 \mathrm{a}$ & $59.0 \pm 4.4 \mathrm{a}$ & $108.7 \pm 28.4 \mathrm{a}$ & $10.5 \pm 1.0 \mathrm{~b}$ & $48.3 \pm 9.7 \mathrm{a}$ & $49.4 \pm 5.8 \mathrm{a}$ & $71.8 \pm 16.5 \mathrm{a}$ & $12.8 \pm 0.9 \mathrm{ab}$ \\
\hline H-SAALA & $51.1 \pm 3.8 \mathrm{ab}$ & $57.7 \pm 3.3 \mathrm{a}$ & $96.9 \pm 18.8 \mathrm{ab}$ & $11.3 \pm 1.1 \mathrm{~b}$ & $45.0 \pm 9.6 \mathrm{a}$ & $48.3 \pm 7.6 \mathrm{a}$ & $77.3 \pm 13.2 \mathrm{a}$ & $11.9 \pm 1.1 \mathrm{ab}$ \\
\hline N-SAALA & $49.0 \pm 4.7 \mathrm{abc}$ & $53.9 \pm 2.8 b$ & $80.6 \pm 15.8 \mathrm{~cd}$ & $11.5 \pm 1.3 \mathrm{ab}$ & $47.7 \pm 8.6 \mathrm{a}$ & $50.5 \pm 7.9 \mathrm{a}$ & $75.4 \pm 16.3 \mathrm{a}$ & $11.8 \pm 1.0 \mathrm{a}$ \\
\hline F-MNBA & $53.1 \pm 7.1 \mathrm{ab}$ & $59.0 \pm 5.1 \mathrm{a}$ & $108.2 \pm 31.3 \mathrm{a}$ & $11.1 \pm 1.0 \mathrm{~b}$ & $43.7 \pm 6.7 \mathrm{a}$ & $50.3 \pm 9.2 \mathrm{a}$ & $81.6 \pm 14.3 \mathrm{a}$ & $11.8 \pm 1.1 \mathrm{~b}$ \\
\hline H-MNBA & $50.2 \pm 5.0 \mathrm{abc}$ & $58.8 \pm 4.3 \mathrm{a}$ & $80.2 \pm 22.9 \mathrm{bcd}$ & $10.8 \pm 0.9 b$ & $42.5 \pm 10.3 \mathrm{a}$ & $50.4 \pm 8.0 \mathrm{a}$ & $67.6 \pm 14.7 \mathrm{a}$ & $12.7 \pm 0.7 \mathrm{ab}$ \\
\hline N-MNBA & $46.0 \pm 7.7 \mathrm{bc}$ & $50.5 \pm 6.5 \mathrm{bc}$ & $69.8 \pm 23.7 \mathrm{~cd}$ & $\begin{array}{l}11.2 \pm 1.1 \mathrm{ab} \\
\text { F-value }\end{array}$ & $41.4 \pm 8.8 \mathrm{a}$ & $51.7 \pm 3.3 \mathrm{a}$ & $78.0 \pm 18.9 \mathrm{a}$ & $12.1 \pm 0.7 \mathrm{ab}$ \\
\hline $\begin{array}{l}\text { Plastic film } \\
\text { mulching }(\mathrm{P})\end{array}$ & 7.1 ** & $7.3 * *$ & $9.8 * *$ & $6.3 * *$ & $0.3 \mathrm{~ns}$ & $0.2 \mathrm{~ns}$ & $1.5 \mathrm{~ns}$ & $0.2 \mathrm{~ns}$ \\
\hline Aeration (A) & $6.8 *$ & $20.0 * *$ & $10.9^{* *}$ & $2.4 \mathrm{~ns}$ & $2.6 \mathrm{~ns}$ & $1.7 \mathrm{~ns}$ & $0.2 \mathrm{~ns}$ & $1.8 \mathrm{~ns}$ \\
\hline $\mathrm{P} \times \mathrm{A}$ & $0.7 \mathrm{~ns}$ & $7.4^{* *}$ & $1.4 \mathrm{~ns}$ & $1.6 \mathrm{~ns}$ & $1.6 \mathrm{~ns}$ & $0.2 \mathrm{~ns}$ & $2.4 \mathrm{~ns}$ & $0.9 \mathrm{~ns}$ \\
\hline
\end{tabular}

Note: F, full film covering; $\mathrm{H}$, half film covering; $\mathrm{N}$, no film covering; $\mathrm{CK}$, no aeration treatment; SAALA subsurface artificial air layer aeration; MNBA, micro-nano-bubble water aeration. Data are presented as the mean \pm standard deviation $(n=9)$. Different lowercase letters in the same column indicate significant differences between treatments (Duncan's test, $p<0.05$ ). ANOVA F-values for main and interaction effects were not significant (ns) or were significant at the $\leq 5 \%\left(^{*}\right)$ and $\leq 1 \%\left({ }^{* *}\right)$ levels. Bold number indicate significant differences at the $\leq 5 \%$ level.
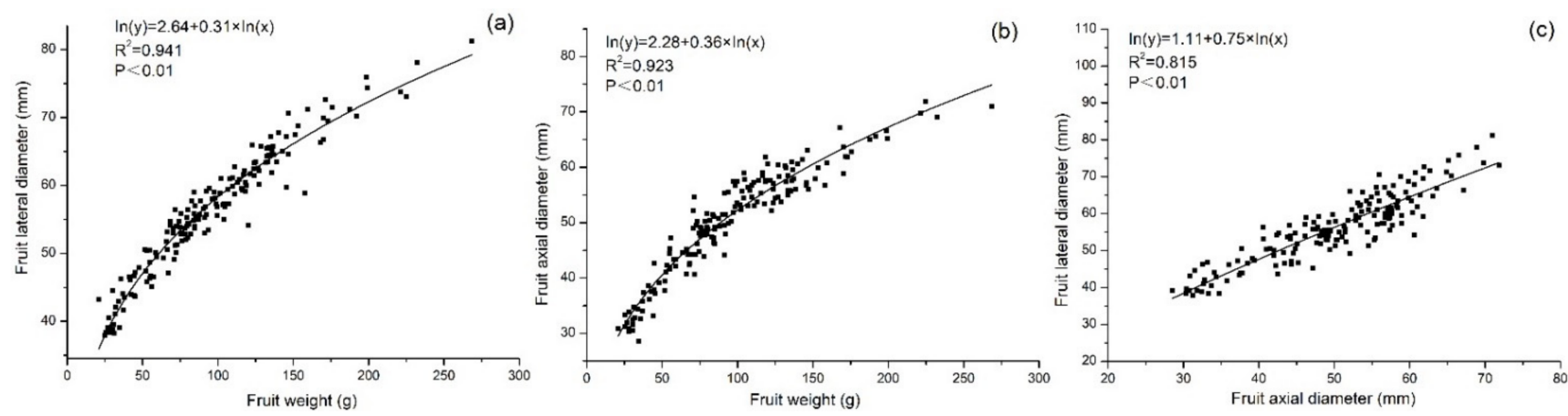

Figure 2. Relationships between (a) fruit weight and width, (b) fruit weight and length (c) fruit width and length.

\subsection{Economic Analysis and Evaluation}

The results of an economic analysis of the soil aeration and PFM treatments in a $100 \mathrm{~m}$-long greenhouse are shown in Table 7 . Two skilled farmers could complete the required PFM work for the whole greenhouse. The local skilled farmer price was $150 \mathrm{CNY}$ day $^{-1}$. The $\mathrm{F}$ and $\mathrm{H}$ film required CNY 300 for additional labour costs. Additional labour costs for the installation of SAALA and MNBA systems were CNY 1000 and 
CNY 150, respectively. The electricity cost for the MNBA treatment was approximately CNY 60. The costs of the F, H, and N PFM were CNY 100, CNY 50, and CNY 0, respectively. We estimated that the depreciation of the SAALA and MNBA systems was CNY 6000 and CNY 1500, respectively. The total yield was calculated according to the average fruit yield per plant (the data are shown in Table 3) multiplied by the number of total plants in the greenhouse (2376 plants greenhouse ${ }^{-1}$ ). The tomato price varied greatly from year to year, and the local average price for the year was $4.5 \mathrm{CNY} \mathrm{kg}^{-1}$. The total income was calculated by multiplying the total yield by 4.5. As shown in Table 7, both the PFM and MNBA treatments increased the income. The calculated maximum additional income was detected in response to the F-MNBA treatment combination. Compared with the N-CK treatment, the F-MNBA treatment combination gained an additional income of CNY 6669 during spring and CNY 2476 during autumn. Notably, all the computations were based on the cost and yield, and measurements of fruit quality did not involve computational processes. If fruit quality is considered, these investments will result in increased profits.

Table 7. Economic analysis of soil aeration and plastic film mulch treatments in a 100-m-long greenhouse.

\begin{tabular}{|c|c|c|c|c|c|c|c|c|c|}
\hline Season & Treatments & $\begin{array}{l}\text { Additional } \\
\text { Labor Cost } \\
\text { for Plastic } \\
\text { Film } \\
\text { Mulch } \\
\text { (CNY) }\end{array}$ & $\begin{array}{l}\text { Additional } \\
\text { Labor Cost } \\
\text { for Soil } \\
\text { Aeration } \\
\text { (CNY) }\end{array}$ & $\begin{array}{c}\text { Additional } \\
\text { Electricity } \\
\text { (CNY) }\end{array}$ & $\begin{array}{l}\text { Plastic } \\
\text { Film } \\
\text { Cost } \\
\text { (CNY) }\end{array}$ & $\begin{array}{c}\text { Depreciation } \\
\text { of the } \\
\text { Equipment } \\
\text { (CNY) }\end{array}$ & $\begin{array}{c}\text { Total Yield } \\
(\mathrm{kg})\end{array}$ & $\begin{array}{l}\text { Total } \\
\text { Income } \\
\text { (CNY) }\end{array}$ & $\begin{array}{c}\text { Additional } \\
\text { Income } \\
\text { Compared } \\
\text { with NCK } \\
\text { (CNY) }\end{array}$ \\
\hline \multirow{9}{*}{ Spring } & F-CK & 300 & 0 & 0 & 100 & 0 & 5733 & 25,799 & 6353 \\
\hline & $\mathrm{H}-\mathrm{CK}$ & 300 & 0 & 0 & 50 & 0 & 4915 & 22,119 & 2723 \\
\hline & N-CK & 0 & 0 & 0 & 0 & 0 & 4232 & 19,046 & 0 \\
\hline & F-SAALA & 300 & 1000 & 0 & 100 & 6000 & 7161 & 32,226 & 5780 \\
\hline & H-SAALA & 300 & 1000 & 0 & 50 & 6000 & 5986 & 26,936 & 540 \\
\hline & N-SAALA & 0 & 1000 & 0 & 0 & 6000 & 5463 & 24,585 & -1461 \\
\hline & F-MNBA & 300 & 150 & 60 & 100 & 1500 & 6183 & 27,825 & 6669 \\
\hline & H-MNBA & 300 & 150 & 60 & 50 & 1500 & 5252 & 23,632 & 2526 \\
\hline & N-MNBA & 0 & 150 & 60 & 0 & 1500 & 4787 & 21,542 & 786 \\
\hline \multirow{9}{*}{ Autumn } & F-CK & 300 & 0 & 0 & 100 & 0 & 4741 & 21,337 & 2357 \\
\hline & $\mathrm{H}-\mathrm{CK}$ & 300 & 0 & 0 & 50 & 0 & 4525 & 20,362 & 1432 \\
\hline & N-CK & 0 & 0 & 0 & 0 & 0 & 4129 & 18,580 & 0 \\
\hline & F-SAALA & 300 & 1000 & 0 & 100 & 6000 & 5969 & 26,861 & 881 \\
\hline & H-SAALA & 300 & 1000 & 0 & 50 & 6000 & 4966 & 22,346 & -3584 \\
\hline & N-SAALA & 0 & 1000 & 0 & 0 & 6000 & 5077 & 22,845 & -2735 \\
\hline & F-MNBA & 300 & 150 & 60 & 100 & 1500 & 5148 & 23,166 & 2476 \\
\hline & H-MNBA & 300 & 150 & 60 & 50 & 1500 & 5238 & 23,570 & 2930 \\
\hline & N-MNBA & 0 & 150 & 60 & 0 & 1500 & 4945 & 22,251 & 1961 \\
\hline
\end{tabular}

Note: F, full film covering; $\mathrm{H}$, half film covering; $\mathrm{N}$, no film covering; $\mathrm{CK}$, no aeration treatment; SAALA, subsurface artificial air layer aeration; MNBA, micro-nano-bubble water aeration.

\section{Discussion}

\subsection{Plant Growth, Yield and Quality of Tomato Fruits under Soil Aeration Treatment}

Tomato fruit yield and quality in terms of flavour, nutrition and shape are affected by various environmental factors, such as light, temperature, water, nutrients, pollutants and soil air, and have been extensively studied [18,34-37]. Zhou et al. [38] reported that plants respond to low $-\mathrm{O}_{2}$ stress by altering their photosynthesis, respiration and biosynthesis of endogenous phytohormones. Previous studies have also shown that root zone hypoxia results in the accumulation of ethanol, ethylene and abscisic acid, accompanied by a reduction in photosynthesis, which affects plant growth and fruit yield [4,39-41]. Tomato is a moderately $\mathrm{O}_{2}$-demanding plant species, and its main roots are distributed within 0-40 cm of the soil surface [42]. Soil aeration has been reported to play an important role in improving soil physical and chemical properties, facilitating tomato plant growth, increasing fruit yield, and maximizing WUE [15,22]. Similar results were obtained in this study; compared with drip irrigation, both MNBA and SAALA improved the dry matter accumulation and yield of tomato plants as well as the flavour, nutrition and shape of the tomato fruits. Other studies have also shown that MNBA increases IWUE, crop yield and quality $[43,44]$. In the present study, compared with the CK, MNBA improved dry matter accumulation (Table 2), and MNBA increased IWUE and the soluble protein content 
in both spring and autumn (Tables 3 and 5). This could be due to hypoxia stress under CK conditions in the soil causing a reduction in plant growth, and the applied MNBA treatments alleviated root zone hypoxia. Liu et al. [18] indicated that MNBA increased IWUE, crop yield and quality, which is in agreement with our results. In general, MNBA provides $\mathrm{O}_{2}$ in the root zone for the growth of tomato plants.

MNBA had positive effects not only on plant growth and yield but also on fruit quality indices (VC, soluble solids, lycopene, soluble protein, soluble sugar, and titratable acid contents as well as the sugar/acid ratio) (Tables 2, 4 and 5). Nevertheless, the results differed during the spring and autumn. Increases in the stem, leaf, root and total dry weight of tomato plants under aeration treatment were found in the spring season, but there were no significant differences in stem or leaf dry weight during autumn (Table 2). Similar results were also found for the flavour and nutrition indices, where the aeration treatments led to a significant increase in titratable acid, VC, and soluble solid contents in the spring season; the increases were not significant during autumn (Tables 4 and 5). A search of the literature revealed no previous reports on MNBA application efficiency during different seasons. Liu's research showed that the ratio of micro/nanobubbles to water in an MNBA system more significant for tomato and cucumber yields during spring than during autumn, but that the oxygenation frequency of MNBA during autumn was more significant [18]. We speculate that this phenomenon may be due to the different weather conditions during spring and autumn. As a general rule, light intensity, temperature, evaporation, humidity, plant bioactivity, and growth rate during spring were higher than those during autumn. Moreover, the $\mathrm{O}_{2}$ demand for plant roots was higher during the spring season than during the autumn season. Therefore, the growing season is short during spring (120 days in this study) and long during autumn (160 days in this study). This also explains why $\mathrm{O}_{2}$ demand became more important during spring than autumn and why soil aeration was more significant during the spring season.

As shown in Table 2, compared with the CK treatment, the SAALA treatment increased the total dry weight during the spring and autumn by $12.99 \%$ and $12.76 \%$, respectively. Fruit yield during the spring and autumn also increased by $25.06 \%$ and $19.53 \%$, respectively (Table 3). The reason for the lower $\mathrm{O}_{2}$ and higher $\mathrm{CO}_{2}$ concentration of the air in the soil pores compared with the outside air is that the air in soil pores is exchanged only through the soil surface under conventional farming practices. Due to the presence of the subsurface air layer, the fluidity and exchange of the air in the soil increased, which increased the $\mathrm{O}_{2}$ concentration in the soil. Furthermore, the subsurface artificial air layer has a unique structure that can prevent water accumulation at the bottom of the plough layer. Thus, compared with the CK treatment, the subsurface SAALA treatment significantly increased the root dry weight and improved other growth indicators (Table 2). Therefore, we speculate that hypoxia stress occurs universally in the CK treatment and that the SAALA treatment can effectively relieve hypoxia stress. The roots grew better in the SAALA treatment than in the other treatment, absorbed more water and nutrients, and affected the yield and quality potential of the tomato plants. Although no previous reports are available on SAALA, other researchers have reported similar observations of soil aeration on plant growth $[45,46]$

\subsection{Plant Growth, Fruit Yield and Quality under PFM Treatment}

The application of PFM for crop management, especially in arid and semiarid regions, has been studied extensively. Previous studies have shown that PFM increases soil water availability and soil temperature, thus increasing various root growth parameters, including root length density, dry weight, surface area and volume, which further increase nutrient and water uptake $[11,47]$. Soil moisture is critical for plants to maintain their growth, especially under high soil-temperature conditions [48]. The ability of tomato plants to absorb water and minerals from the soil is attributed primarily to their extensive root system. In the present study, root dry weight markedly increased under PFM treatment during both spring and autumn in tomato (Table 2). Moreover, the total dry weight significantly increased in response to PFM. It was demonstrated that PFM could effectively 
improve root growth as well as water and nutrient uptake, and could, to some extent, affect the performance of plant growth [49]. The conclusion of that study is consistent with our results. We also found that the total dry weight of the plants was significantly higher under the PFM treatment than under the $\mathrm{N}$ treatment in both the MNBA and SAALA treatments (Table 2). This finding suggests that PFM is practical and adaptable under aeration. Although this study did not measure the physiological indices of plants, other studies have shown that PFM increases the topsoil temperature and moisture content as well as the WUE, all of which have positive effects on photosynthesis and fruit yield [11,50]. Therefore, we hypothesized that an increased net photosynthesis rate is one of the main mechanisms by which PFM increases plant growth. In addition, soil enzyme and microbial activities have been shown to be affected by soil PFM treatments [30]. All these changes could interact and affect nutrient cycling and nutrient bioaccessibility, affecting plant growth [51].

PFM significantly increased IWUE, as suggested by Zhang et al., [52] and Fan et al., [53]. In the present study, IWUE significantly increased in response to PFM during the spring but not during autumn (Table 3). We speculate that the relatively high atmospheric and soil temperatures during the spring are accompanied by increased evaporation and transpiration. PFM restricts evaporation, thereby improving the IWUE substantially. In contrast, evaporation is relatively low during autumn. Therefore, no significant differences in IWUE were observed between the PFM and $\mathrm{N}$ treatments. These results are consistent with those of Díaz-Pérez [54], who reported that plastic mulch affected plant growth, and the effect of mulch was more dramatic in the spring season than in the autumn season.

In addition to fruit yield, fruit quality was also affected by the PFM treatment. It is well-known that soluble sugar and titratable acid contents affect fruit flavour. PFM resulted in a relatively low accumulation of soluble sugar and titratable acid contents in the fruits in both the spring and autumn (Table 4), which implied that the PFM treatment decreased the flavour indices of the tomato fruits. Previous studies have shown that a high soil water content leads to a decrease in both fruit flavour and nutritional quality $[36,55]$. We postulated that PFM increases soil moisture [11] and that high soil moisture leads to a decrease in soluble solids and soluble protein contents in the fruits. Furthermore, this study showed that the soluble solids content, lycopene content and shape indices of the tomato fruits significantly increased in response to the PFM treatment during the spring season (Tables 5 and 6). Lycopene is specifically important for the human diet and has been linked to a reduced risk of prostate cancer and heart disease [56]. Xia's research [57] suggested that PFM treatment has a positive impact on lycopene content, which is consistent with our results.

\subsection{Correlations between Plant Growth, Fruit Yield and Fruit Quality under Soil Aeration and PFM Treatments}

A large number of studies have shown that plant performance and fruit yield promote soil physicochemical properties, such as soil moisture, temperature, air concentration, $\mathrm{pH}$, and microbial activity $[15,16,58]$. However, no previous studies have examined the specific correlations between plant performance and quality under soil aeration and PFM. A previous study showed that a low $\mathrm{O}_{2}$ supply can decrease root and shoot growth of tomato while decreasing root total respiration and hydrolytic ATPase activities [59]. Figure 3 shows the correlations between tomato plant performance, fruit flavour, fruit nutrition and fruit shape indicators. Root dry weight was significantly positively correlated with fruit weight during both the spring (Figure 3a) and autumn (Figure 3b) $(p<0.01)$. Furthermore, fruit weight was significantly positively correlated with VC and soluble protein contents. Overall, our results indicate that both soil aeration and PFM increased dry matter accumulation, plant growth and quality of tomato. 


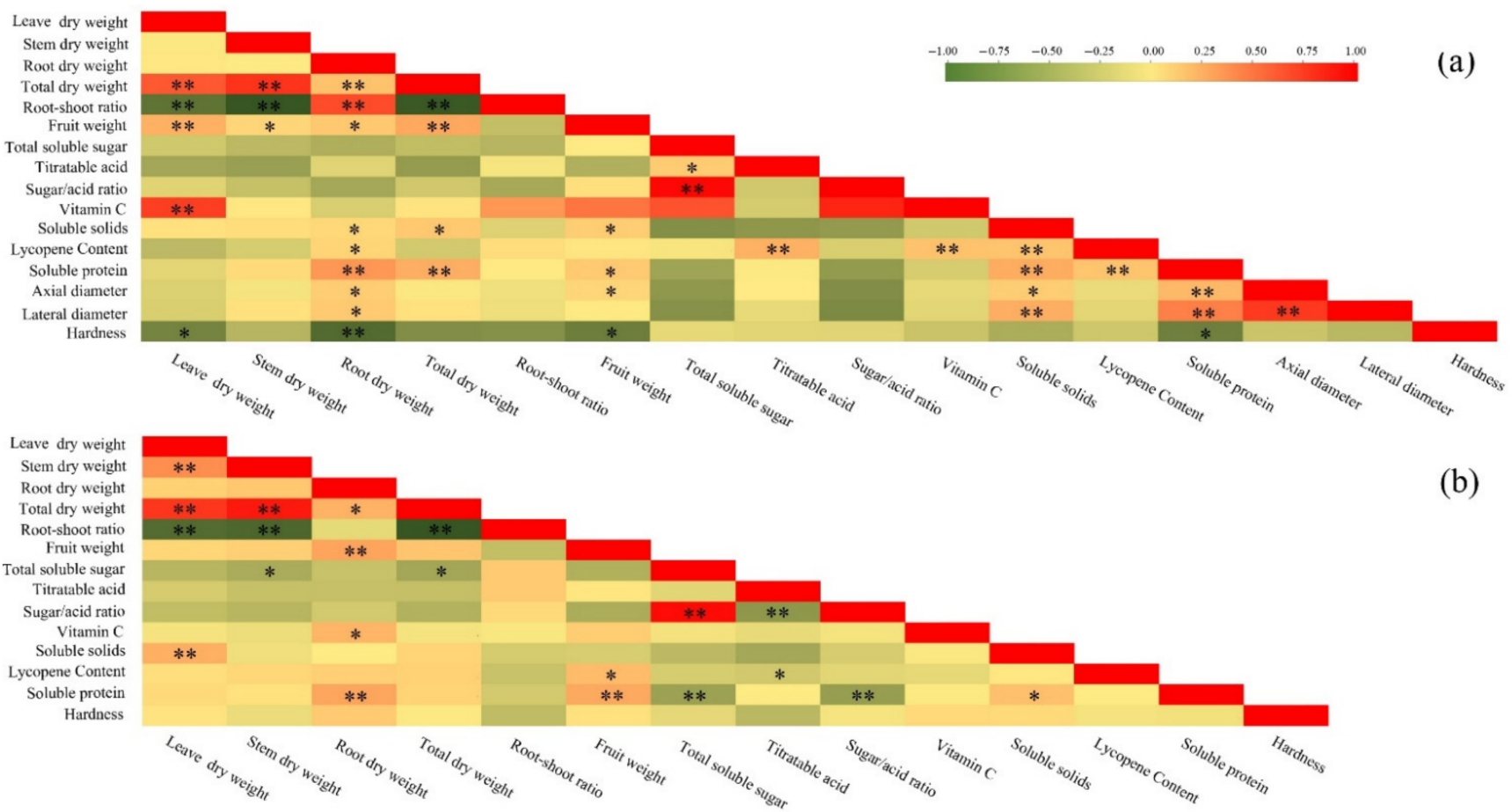

Figure 3. Pearson correlation between growth and quality parameters of tomato during spring (a) and autumn (b) under soil aeration and plastic film mulch treatments. ${ }^{*}$ Significant at the 5\%; ** Significant at the 1\% (2-tailed).

\section{Conclusions}

The results of this experiment showed that it is feasible to configure the installation and operation of commercially available PFM and SAALA and MNBA systems to regulate the rootzone environment and indirectly influence tomato plant growth, yield and fruit quality. Both MNBA and SAALA increased the plant dry weight, fruit yield and IWUE. In comparison with the no aeration treatment, the MNBA treatment increased dry matter accumulation, fruit yield, IWUE, lycopene content and soluble protein content during the spring season by $7.1 \%, 9.0 \%, 7.1 \%, 6.2 \%$ and $16.2 \%$, respectively, while those during autumn increased by $4.0 \%, 14.4 \%, 4.0 \%, 3.8 \%$ and $1.7 \%$, respectively. During the spring season, SAALA increased dry matter accumulation, fruit yield, IWUE, lycopene content and soluble protein content by $13.0 \%, 25.1 \%, 13.0 \%, 2.9 \%$ and $15.1 \%$, respectively, while those during autumn increased by $12.8 \%, 19.5 \%, 12.8 \%, 5.2 \%$ and $4.1 \%$, respectively. The total dry weight, fruit yield and IWUE increased in response to the PFM treatment. Nevertheless, PFM decreased the content of soluble protein. The increased yield in response to soil aeration during autumn was more significant than that during the spring, and the increased yield in response to PFM during the spring was more significant than that during autumn. Considering the effects of cost, efficiency and benefits, the greatest economic benefits were obtained in the MNBA and F PFM treatment combination. These findings suggest that soil aeration and PFM treatment cause significant changes in soil physical and chemical properties, which subsequently affect plant performance and fruit quality.

Author Contributions: Y.L. and Z.Z. conceived the idea for this study. Y.L. and M.Z. supervised the project. J.W. collected the samples. Y.L., M.Z. and J.W. performed the composting experiment. M.Z. and Y.L. performed laboratory work. Y.L. and M.Z. conducted data analysis. Y.L. and M.Z. wrote and revised the manuscript. All authors have read and agreed to the published version of the manuscript. 
Funding: This work is supported jointly by Natural Science Foundation of China (No. 41831284, No. 41807041), Science and Technology Program of Xi'an (20193052YF040NS040), Natural Science Foundation of Guangdong Province (No. 2018A0303130149), Science and Technology Program of Guangzhou (No. 20181002SF0530), Fundamental Research Funds for the Central Universities (GK202103129), and the Program of Introducing Talents of Discipline to Universities (B16011). We are grateful for the helpful comments of the anonymous reviewers.

Institutional Review Board Statement: Not applicable.

Informed Consent Statement: Not applicable.

Data Availability Statement: The datasets used and/or analysed during the current study are available from the corresponding author on reasonable request.

Conflicts of Interest: The authors declare no conflict of interest.

\section{Appendix A}

Table A1. Main technical parameters of the micro-nano generator.

\begin{tabular}{cccc}
\hline Indicators & Parameters & Indicators & Parameters \\
\hline Operating voltage & $220 \mathrm{~V} \sim 50 \mathrm{~Hz}$ & Power & $300 \mathrm{~W}$ \\
Working pressure & $0-0.30 \mathrm{Mpa}$ & Bubble rise velocity & $10-15 \mathrm{~mm} / \mathrm{s}$ \\
Bubble size & $700 \mathrm{~nm}-2 \mu \mathrm{m}$ & Air content & $0-90 \%$ \\
\hline
\end{tabular}

Table A2. Irrigation date and amount during both growing seasons.

\begin{tabular}{|c|c|c|c|}
\hline \multicolumn{2}{|c|}{ Spring } & \multicolumn{2}{|c|}{ Autumn } \\
\hline Irrigation Date & $\begin{array}{l}\text { Irrigation Amount } \\
(\mathrm{mm})\end{array}$ & Irrigation Date & $\begin{array}{c}\text { Irrigation Amount } \\
(\mathrm{mm})\end{array}$ \\
\hline 9 April 2019 & 26.23 & 4 September 2019 & 25.80 \\
\hline 14 April 2019 & 9.41 & 9 September 2019 & 25.88 \\
\hline 19 April 2019 & 25.38 & 14 September 2019 & 5.07 \\
\hline 24 April 2019 & 13.07 & 19 September 2019 & 4.13 \\
\hline 29 April 2019 & 13.84 & 24 September 2019 & 16.61 \\
\hline 4 May 2019 & 17.75 & 29 September 2019 & 19.39 \\
\hline 9 May 2019 & 22.89 & 4 October 2019 & 9.16 \\
\hline 14 May 2019 & 10.45 & 9 October 2019 & 5.87 \\
\hline 19 May 2019 & 16.62 & 14 October 2019 & 7.21 \\
\hline 24 May 2019 & 33.16 & 19 October 2019 & 8.78 \\
\hline 29 May 2019 & 26.37 & 24 October 2019 & 6.47 \\
\hline 3 June 2019 & 22.84 & 29 October 2019 & 10.58 \\
\hline 8 June 2019 & 24.29 & 3 November 2019 & 9.15 \\
\hline 13 June 2019 & 20.29 & 8 November 2019 & 6.88 \\
\hline 18 June 2019 & 21.68 & 13 November 2019 & 7.11 \\
\hline 23 June 2019 & 6.71 & 18 November 2019 & 8.77 \\
\hline 28 June 2019 & 15.51 & 23 November 2019 & 8.55 \\
\hline 3 July 2019 & 20.65 & 28 November 2019 & 5.26 \\
\hline 8 July 2019 & 35.05 & 3 December 2019 & 6.34 \\
\hline 13 July 2019 & 26.34 & 8 December 2019 & 8.99 \\
\hline 15 July 2019 & 15.12 & 13 December 2019 & 10.68 \\
\hline \multirow[t]{8}{*}{ Total irrigation amount } & 423.64 & 18 December 2019 & 7.06 \\
\hline & & 23 December 2019 & 7.66 \\
\hline & & 28 December 2019 & 6.78 \\
\hline & & 2 January 2020 & 7.05 \\
\hline & & 7 January 2020 & 6.64 \\
\hline & & 12 January 2020 & 5.64 \\
\hline & & 17 January 2020 & 4.32 \\
\hline & & $\begin{array}{c}\text { Total irrigation } \\
\text { amount }\end{array}$ & 261.83 \\
\hline
\end{tabular}


(a)
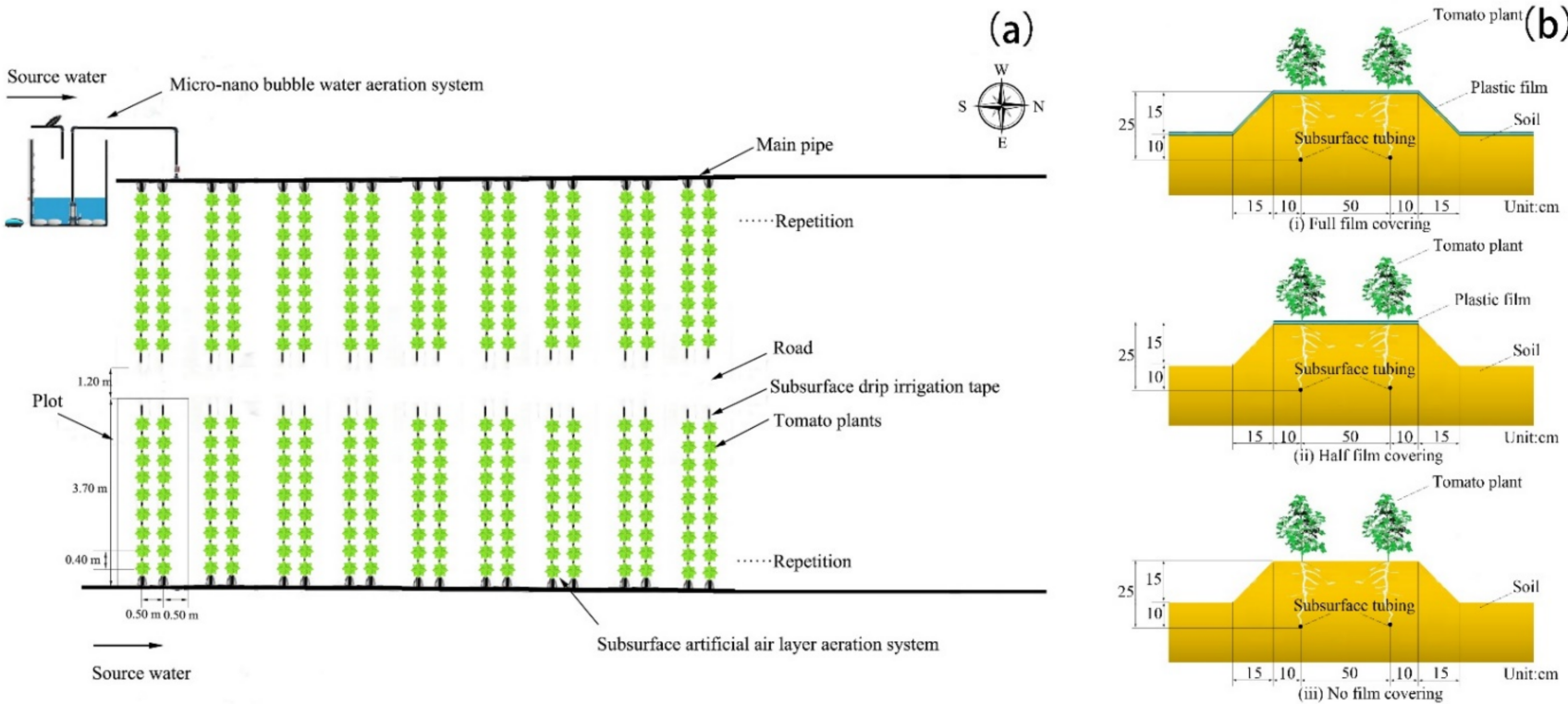

Experimental arrangement of aeration treatments

Experimental arrangement of plastic film mulch treatments

Figure A1. (a) Experimental arrangement of aeration and (b) plastic film mulch treatments.

\section{References}

1. Ben-Noah, I.; Friedman, S.P. Aeration of clayey soils by injecting air through subsurface drippers: Lysimetric and field experiments. Agric. Water Manag. 2016, 176, 222-233. [CrossRef]

2. Oliveira, H.C.; Freschi, L.; Sodek, L. Nitrogen metabolism and translocation in soybean plants subjected to root oxygen deficiency. Plant Physiol. Biochem. 2013, 66, 141-149. [CrossRef] [PubMed]

3. Gao, H.; Jia, Y.; Guo, S.; Lv, G.; Wang, T.; Juan, L. Exogenous calcium affects nitrogen metabolism in root-zone hypoxia-stressed muskmelon roots and enhances short-term hypoxia tolerance. J. Plant Physiol. 2011, 168, 1217-1225. [CrossRef] [PubMed]

4. Bai, T.; Li, C.; Li, C.; Liang, D.; Ma, F. Contrasting hypoxia tolerance and adaptation in Malus species is linked to differences in stomatal behavior and photosynthesis. Physiol. Plant 2013, 147, 514-523. [CrossRef] [PubMed]

5. Li, C.; Bai, T.; Ma, F.; Han, M. Hypoxia tolerance and adaptation of anaerobic respiration to hypoxia stress in two Malus species. Sci. Hortic. 2010, 124, 274-279. [CrossRef]

6. Kläring, H.; Zude, M. Sensing of tomato plant response to hypoxia in the root environment. Sci. Hortic. 2009, 122, 17-25. [CrossRef]

7. Fukao, T.; Bailey-Serres, J. Plant responses to hypoxia-is survival a balancing act? Trends Plant Sci. 2004, 9, 449-456. [CrossRef]

8. Kader, M.A.; Senge, M.; Mojid, M.A.; Ito, K. Recent advances in mulching materials and methods for modifying soil environment. Soil. Tillage Res. 2017, 168, 155-166. [CrossRef]

9. Jia, Q.; Sun, L.; Ali, S.; Zhang, Y.; Liu, D.; Kamran, M.; Zhang, P.; Jia, Z.; Ren, X. Effect of planting density and pattern on maize yield and rainwater use efficiency in the Loess Plateau in China. Agric. Water Manag. 2018, 202, 19-32. [CrossRef]

10. Díaz-Pérez, J.C.; Gitaitis, R.; Mandal, B. Effects of plastic mulches on root zone temperature and on the manifestation of tomato spotted wilt symptoms and yield of tomato. Sci. Hortic. 2007, 114, 90-95. [CrossRef]

11. Zhang, X.; Yang, L.; Xue, X.; Kamran, M.; Ahmad, I.; Dong, Z.; Liu, T.; Jia, Z.; Zhang, P.; Han, Q. Plastic film mulching stimulates soil wet-dry alternation and stomatal behavior to improve maize yield and resource use efficiency in a semi-arid region. Field Crop Res. 2019, 233, 101-113. [CrossRef]

12. Niu, L.; Yan, Y.; Hou, P.; Bai, W.; Zhao, R.; Wang, Y.; Li, S.; Du, T.; Zhao, M.; Song, J.; et al. Influence of plastic film mulching and planting density on yield, leaf anatomy, and root characteristics of maize on the Loess Plateau. Crop J. 2020, 8, 548-564. [CrossRef]

13. Mo, F.; Wang, J.; Zhou, H.; Luo, C.; Zhang, X.; Li, X.; Li, F.; Xiong, L.; Kavagi, L.; Nguluu, S.N.; et al. Ridge-furrow plastic-mulching with balanced fertilization in rainfed maize (Zea mays L.): An adaptive management in east African Plateau. Agric. For. Meteorol. 2017, 236, 100-112. [CrossRef]

14. Cheng, Z.; Chen, Y.; Zhang, F. Effect of reclamation of abandoned salinized farmland on soil bacterial communities in arid northwest China. Sci. Total Environ. 2018, 630, 799-808. [CrossRef]

15. Li, Y.; Niu, W.; Wang, J.; Liu, L.; Zhang, M.; Xu, J. Effects of artificial soil aeration volume and frequency on soil enzyme activity and microbial abundance when cultivating greenhouse tomato. Soil Sci. Soc. Am. J. 2016, 80, 1208. [CrossRef]

16. Chen, X.M.; Dhungel, J.; Bhattarai, S.P.; Torabi, M.; Pendergast, L.; Midmore, D.J. Impact of oxygation on soil respiration, yield and water use efficiency of three crop species. J. Plant Ecol. 2011, 4, 236-248. [CrossRef]

17. Shahien, M.M.; Abuarab, M.E.; Magdy, E. Root aeration improves yield and water use efficiency of irrigated potato in sandy clay loam soil. Int. J. Adv. Res. 2014, 2, 310-320. 
18. Liu, Y.; Zhou, Y.; Wang, T.; Pan, J.; Zhou, B.; Muhammad, T.; Zhou, C.; Li, Y. Micro-nano bubble water oxygation: Synergistically improving irrigation water use efficiency, crop yield and quality. J. Clean Prod. 2019, 222, 835-843. [CrossRef]

19. Bhattarai, S.P.; Huber, S.; Midmore, D.J. Aerated subsurface irrigation water gives growth and yield benefits to Zucchini, vegetable soybean and cotton in heavy clay soils. Ann. Appl. Biol. 2004, 144, 285-298. [CrossRef]

20. Li, Y.; Niu, W.; Zhang, M.; Wang, J.; Zhang, Z. Artificial soil aeration increases soil bacterial diversity and tomato root performance under greenhouse conditions. Land Degrad Dev. 2020, 31, 1443-1461. [CrossRef]

21. Shahien, M.M.; Abuarab, M.E.; Magdy, E. Root aeration improves yield and water use efficiency of tomato in heavy clay and saline soils. Sci. Hortic. 2006, 108, 278-288.

22. Dhungel, J.; Bhattarai, S.P.; Midmore, D.J. Aerated water irrigation (oxygation) benefits to pineapple yield, water use efficiency and crop health. Adv. Hortic. Sci. 2012, 26, 3-16.

23. Friedman, S.P.; Naftaliev, B. A survey of the aeration status of drip-irrigated orchards. Agric. Water Manag. 2012, $115,132-147$. [CrossRef]

24. Li, Y.; Niu, W.; Xu, J.; Wang, J.; Zhang, M.; Wang, L. Root morphology of greenhouse produced muskmelon under sub-surface drip irrigation with supplemental soil aeration. Sci. Hortic. 2016, 201, 287-294. [CrossRef]

25. Pendergast, L.; Bhattarai, S.P.; Midmore, D.J. Benefits of oxygation of subsurface drip-irrigation water for cotton in a Vertosol. Crop Pasture Sci. 2013, 64, 1171-1181. [CrossRef]

26. Bhattarai, S.P.; Midmore, D.J.; Pendergast, L. Yield, water-use efficiencies and root distribution of soybean, chickpea and pumpkin under different subsurface drip irrigation depths and oxygation treatments in vertisols. Irrig. Sci. 2008, 26, 439-450. [CrossRef]

27. Goorahoo, D.; Carstensen, G.; Zoldoske, D. Using air in sub-surface drip irrigation (SDI) to increase yields in bell peppers. Int Water Irrig. 2002, 22, 39-42.

28. Lee, J.; Lee, B.; Kang, J.; Bae, J.; Ku, Y.; Gorinstein, S.; Lee, J. Effect of root zone aeration on the growth and bioactivity of cucumber plants cultured in perlite substrate. Biologia 2014, 69, 610-617. [CrossRef]

29. Nangare, D.D.; Singh, Y.; Kumar, P.S.; Minhas, P.S. Growth, fruit yield and quality of tomato (Lycopersicon esculentum Mill.) as affected by deficit irrigation regulated on phenological basis. Agric. Water Manag. 2016, 171, 73-79. [CrossRef]

30. Zhang, X.; You, S.; Tian, Y.; Li, J. Comparison of plastic film, biodegradable paper and bio-based film mulching for summer tomato production: Soil properties, plant growth, fruit yield and fruit quality. Sci. Hortic. 2019, 249, 38-48. [CrossRef]

31. Gao, J.F. Experimental Guidance for Plant Physiology; Higher Education Press: Beijing, China, 2006.

32. Zhang, L.F.; Ding, X.L. Establishment of a new lycopene determination method. Food Ferment. Ind. 2001, $27,51-55$.

33. Gould, W.A. Tomato Production, Processing, and Technology, 3rd ed.; CTI Publications: Baltimore, MD, USA, 1992.

34. Kim, H.; Yang, T.; Choi, S.; Wang, Y.; Lin, M.; Liceaga, A.M. Supplemental intracanopy far-red radiation to red LED light improves fruit quality attributes of greenhouse tomatoes. Sci. Hortic. 2020, 261, 108985. [CrossRef]

35. Watanabe, M.; Ohta, Y.; Licang, S.; Motoyama, N.; Kikuchi, J. Profiling contents of water-soluble metabolites and mineral nutrients to evaluate the effects of pesticides and organic and chemical fertilizers on tomato fruit quality. Food Chem. 2015, 169, 387-395. [CrossRef]

36. Lu, J.; Shao, G.; Cui, J.; Wang, X.; Keabetswe, L. Yield, fruit quality and water use efficiency of tomato for processing under regulated deficit irrigation: A meta-analysis. Agric. Water Manag. 2019, 222, 301-312. [CrossRef]

37. Farneti, B.; Schouten, R.E.; Qian, T.; Dieleman, J.A.; Tijskens, L.M.M.; Woltering, E.J. Woltering, Greenhouse climate control affects postharvest tomato quality. Postharvest Biol. Technol. 2013, 86, 354-361. [CrossRef]

38. Zhou, W.; Chen, F.; Meng, Y.; Chandrasekaran, U.; Luo, X.; Yang, W.; Shu, K. Plant waterlogging/flooding stress responses: From seed germination to maturation. Plant Physiol. Biochem. 2020, 148, 228-236. [CrossRef]

39. Armstrong, W.; Beckett, P.M.; Colmer, T.D.; Setter, T.L.; Greenway, H. Tolerance of roots to low oxygen: 'Anoxic'cores, the phytoglobin-nitric oxide cycle, and energy or oxygen sensing. J. Plant Physiol. 2019, 239, 92-108. [CrossRef]

40. Rajapakse, N.C.; He, C.; Cisneros-Zevallos, L.; Davies, F.T. Hypobaria and hypoxia affects growth and phytochemical contents of lettuce. Sci. Hortic. 2009, 122, 171-178. [CrossRef]

41. Saher, S.; Fernández-García, N.; Piqueras, A.; Hellín, E.; Olmos, E. Reducing properties, energy efficiency and carbohydrate metabolism in hyperhydric and normal carnation shoots cultured in vitro: A hypoxia stress? Plant Physiol. Biochem. 2005, 43, 573-582. [CrossRef]

42. Zheng, Y.; Wang, L.; Dixon, M. An upper limit for elevated root zone dissolved oxygen concentration for tomato. Sci. Hortic. 2007, 113, 162-165. [CrossRef]

43. Zhou, Y.; Zhou, B.; Xu, F.; Muhammad, T.; Li, Y. Appropriate dissolved oxygen concentration and application stage of micro-nano bubble water oxygation in greenhouse crop plantation. Agric. Water Manag. 2019, 223, 105713. [CrossRef]

44. Zhou, Y.; Bastida, F.; Zhou, B.; Sun, Y.; Gu, T.; Li, S.; Li, Y. Soil fertility and crop production are fostered by micro-nano bubble irrigation with associated changes in soil bacterial community. Soil Biol. Biochem. 2020, 141, 107663. [CrossRef]

45. Pendergast, L.; Bhattarai, S.P.; Midmore, D.J. Evaluation of aerated subsurface drip irrigation on yield, dry weight partitioning and water use efficiency of a broad-acre chickpea (Cicer arietinum, L.) in a vertosol. Agric. Water Manag. 2019, 217, 38-46. [CrossRef]

46. Cui, B.; Niu, W.; Du, Y.; Zhang, Q. Response of yield and nitrogen use efficiency to aerated irrigation and $\mathrm{N}$ application rate in greenhouse cucumber. Sci. Hortic. 2020, 265, 109220. [CrossRef]

47. Gu, Y.; Han, C.; Fan, J.; Shi, X.; Kong, M.; Shi, X.; Siddique, K.H.M.; Zhao, Y.; Li, F. Alfalfa forage yield, soil water and P availability in response to plastic film mulch and P fertilization in a semiarid environment. Field Crop Res. 2018, 215, 94-103. [CrossRef] 
48. Lobell, D.B.; Hammer, G.L.; Chenu, K.; Zheng, B.; Mclean, G.; Chapman, S.C. The shifting influence of drought and heat stress for crops in northeast Australia. Global Change Biol. 2015, 21, 4115-4127. [CrossRef]

49. Gu, X.; Cai, H.; Du, Y.; Li, Y. Effects of film mulching and nitrogen fertilization on rhizosphere soil environment, root growth and nutrient uptake of winter oilseed rape in northwest China. Soil. Tillage Res. 2019, 187, 194-203. [CrossRef]

50. Ali, S.; Xu, Y.; Jia, Q.; Ma, X.; Ahmad, I.; Adnan, M.; Gerard, R.; Ren, X.; Zhang, P.; Cai, T.; et al. Interactive effects of plastic film mulching with supplemental irrigation on winter wheat photosynthesis, chlorophyll fluorescence and yield under simulated precipitation conditions. Agric. Water Manag. 2018, 207, 1-14. [CrossRef]

51. Dungait, J.A.J.; Hopkins, D.W.; Gregory, A.S.; Whitmore, A.P. Soil organic matter turnover is governed by accessibility not recalcitrance. Global Change Biol. 2012, 18, 1781-1796. [CrossRef]

52. Zhang, Y.; Wang, J.; Gong, S.; Xu, D.; Sui, J.; Wu, Z.; Mo, Y. Effects of film mulching on evapotranspiration, yield and water use efficiency of a maize field with drip irrigation in Northeastern China. Agric. Water Manag. 2018, 205, 90-99. [CrossRef]

53. Fan, Y.; Ding, R.; Kang, S.; Hao, X.; Du, T.; Tong, L.; Li, S. Plastic mulch decreases available energy and evapotranspiration and improves yield and water use efficiency in an irrigated maize cropland. Agric. Water Manag. 2017, 179, 122-131. [CrossRef]

54. Díaz-Pérez, J.C. Root zone temperature, plant growth, yield of broccoli [Brassica oleracea (Plenck) var. italica] as affected by plastic film mulches. Sci. Hortic. 2009, 123, 156-163.

55. Du, Y.; Cao, H.; Liu, S.; Gu, X.; Cao, Y. Response of yield, quality, water and nitrogen use efficiency of tomato to different levels of water and nitrogen under drip irrigation in Northwestern China. J. Integr. Agric. 2017, 16, 1153-1161. [CrossRef]

56. Tan, H.; Thomas-Ahner, J.M.; Grainger, E.M.; Wan, L.; Francis, D.M.; Schwartz, S.J.; Erdman, J.W.; Clinton, S.K. Tomato-based food products for prostate cancer prevention: What have we learned? Cancer Metastasis Rev. 2010, 29, 553-568. [CrossRef]

57. Xia, D.; Li, J.; Wang, G.; Jiang, F.; Wu, Z. Effects of different mulching methods on soil fertility and the yield and quality of tomato. Chin. J. Ecol. 2014, 33, 1826-1832.

58. Kuklik, V.; Hoang, T.D. Soil moisture regimes under point irrigation. Agric. Water Manag. 2014, 134, 42-49. [CrossRef]

59. Shi, K.; Hu, W.; Dong, D.; Zhou, Y.; Yu, J. Low $\mathrm{O}_{2}$ supply is involved in the poor growth in root-restricted plants of tomato (Lycopersicon esculentum Mill.). Environ. Exp. Bot. 2007, 61, 181-189. [CrossRef] 